\title{
ALMA Reveals Potential Evidence for Spiral Arms, Bars, and Rings in High-redshift Submillimeter Galaxies
}

Hodge, J. A.; Smail, I.; Walter, F.; da Cunha, E.; Swinbank, A. M.; Rybak, M.; Venemans, B.; Brandt, W. N.; Rivera, G. Calistro; Chapman, S. C.

Total number of authors:

22

Published in:

Astrophysical Journal

Link to article, DOI:

10.3847/1538-4357/ab1846

Publication date:

2019

Document Version

Publisher's PDF, also known as Version of record

Link back to DTU Orbit

Citation $(A P A)$ :

Hodge, J. A., Smail, I., Walter, F., da Cunha, E., Swinbank, A. M., Rybak, M., Venemans, B., Brandt, W. N., Rivera, G. C., Chapman, S. C., Chen, C-C., Cox, P., Dannerbauer, H., Decarli, R., Greve, T. R., Knudsen, K. K., Menten, K. M., Schinnerer, E., Simpson, J. M., ... Weiss, A. (2019). ALMA Reveals Potential Evidence for Spiral Arms, Bars, and Rings in High-redshift Submillimeter Galaxies. Astrophysical Journal, 876(2), [130]. https://doi.org/10.3847/1538-4357/ab1846 


\title{
ALMA Reveals Potential Evidence for Spiral Arms, Bars, and Rings in High-redshift Submillimeter Galaxies
}

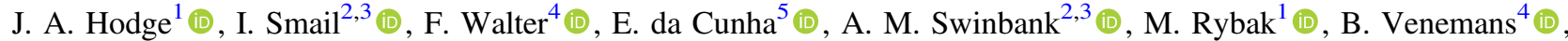 \\ W. N. Brandt ${ }^{6,7,8}\left(\right.$ (i) , G. Calistro Rivera ${ }^{1}$, S. C. Chapman ${ }^{9}$, Chian-Chou Chen ${ }^{10}$ (i), P. Cox ${ }^{11}$, H. Dannerbauer ${ }^{12,13}$ (i), R. Decarli ${ }^{14}$ (i),

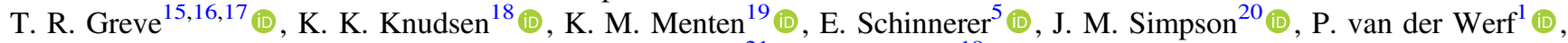 \\ J. L. Wardlow ${ }^{21}$, and A. Weiss ${ }^{19}$ (i) \\ ${ }^{1}$ Leiden Observatory, Leiden University, P.O. Box 9513, 2300 RA Leiden, The Netherlands; hodge@strw.leidenuniv.nl \\ ${ }^{2}$ Centre for Extragalactic Astronomy, Department of Physics, Durham University, South Road, Durham, DH1 3LE, UK \\ ${ }^{3}$ Institute for Computational Cosmology, Durham University, South Road, Durham, DH1 3LE, UK \\ ${ }^{4}$ Max-Planck Institut für Astronomie, Königstuhl 17, D-69117 Heidelberg, Germany
${ }^{5}$ Research School of Astronomy and Astrophysics, Australian National University, Canberra, ACT 2611, Australia \\ ${ }^{6}$ Department of Astronomy \& Astrophysics, 525 Davey Lab, The Pennsylvania State University, University Park, PA 16802, USA \\ ${ }^{7}$ Institute for Gravitation and the Cosmos, The Pennsylvania State University, University Park, PA 16802, USA \\ ${ }^{8}$ Department of Physics, 104 Davey Laboratory, The Pennsylvania State University, University Park, PA 16802, USA \\ ${ }^{9}$ Department of Physics and Atmospheric Science, Dalhousie University, 6310 Coburg Road, Halifax, NS B3H 4R2, Canada \\ ${ }^{10}$ European Southern Observatory, Karl Schwarzschild Straße 2, Garching, Germany \\ ${ }^{11}$ Institut d'Astrophysique de Paris, CNRS, UMR 7095, 98bis boulevard Arago, F-75014, Paris, France \\ ${ }^{12}$ Instituto de Astrofísica de Canarias, Vía Láctea s/n, E-38205, La Laguna, Tenerife, Spain \\ ${ }^{13}$ Universidad de La Laguna, Dpto. Astrofsica, E-38206 La Laguna, Tenerife, Spain \\ ${ }^{14}$ INAF/Osservatorio di Astrofisica e Scienza dello Spazio di Bologna, Via Gobetti 93/3, I-40129 Bologna, Italy \\ ${ }^{15}$ Cosmic Dawn Center (DAWN), DTU-Space, Technical University of Denmark, Elektrovej 327, DK-2800 Kgs. Lyngby, Denmark \\ ${ }_{17}^{16}$ Niels Bohr Institute, University of Copenhagen, Juliane Maries Vej 30, DK-2100 Copenhagen Ø, Denmark \\ ${ }^{17}$ University College London, Department of Physics \& Astronomy, Gower Street, London, WC1E 6BT, UK \\ ${ }^{18}$ Department of Space, Earth and Environment, Chalmers University of Technology, Onsala Space Observatory, SE-43992 Onsala, Sweden \\ ${ }^{19}$ Max-Planck Institut für Radioastronomie, Auf dem Hügel 69, D-53121 Bonn, Germany \\ ${ }^{20}$ Academia Sinica Institute of Astronomy and Astrophysics, No. 1, Sec. 4, Roosevelt Road, Taipei 10617, Taiwan \\ ${ }^{21}$ Physics Department, Lancaster University, Lancaster, LA1 4 YB, UK \\ Received 2018 October 29; revised 2019 April 5; accepted 2019 April 9; published 2019 May 13
}

\begin{abstract}
We present subkiloparsec-scale mapping of the $870 \mu \mathrm{m}$ ALMA continuum emission in six luminous $\left(L_{\mathrm{IR}} \sim 5 \times 10^{12} L_{\odot}\right)$ submillimeter galaxies (SMGs) from the ALESS survey of the Extended Chandra Deep Field South. Our high-fidelity 0 !"07-resolution imaging $(\sim 500 \mathrm{pc})$ reveals robust evidence for structures with deconvolved sizes of $\lesssim 0.5-1 \mathrm{kpc}$ embedded within (dominant) exponential dust disks. The large-scale morphologies of the structures within some of the galaxies show clear curvature and/or clump-like structures bracketing elongated nuclear emission, suggestive of bars, star-forming rings, and spiral arms. In this interpretation, the ratio of the "ring" and "bar" radii $(1.9 \pm 0.3)$ agrees with that measured for such features in local galaxies. These potential spiral/ring/bar structures would be consistent with the idea of tidal disturbances, with their detailed properties implying flat inner rotation curves and Toomre-unstable disks $(Q<1)$. The inferred one-dimensional velocity dispersions $\left(\sigma_{\mathrm{r}} \lesssim 70-160 \mathrm{~km} \mathrm{~s}^{-1}\right)$ are marginally consistent with the limits implied if the sizes of the largest structures are comparable to the Jeans length. We create maps of the star formation rate density $\left(\Sigma_{\mathrm{SFR}}\right)$ on $\sim 500 \mathrm{pc}$ scales and show that the SMGs are able to sustain a given (galaxy-averaged) $\Sigma_{\mathrm{SFR}}$ over much larger physical scales than local (ultra)luminous infrared galaxies. However, on 500 pc scales, they do not exceed the Eddington limit set by radiation pressure on dust. If confirmed by kinematics, the potential presence of nonaxisymmetric structures would provide a means for net angular momentum loss and efficient star formation, helping to explain the very high star formation rates measured in SMGs.
\end{abstract}

Key words: galaxies: evolution - galaxies: formation - galaxies: high-redshift - galaxies: starburst - submillimeter: galaxies

\section{Introduction}

At the peak of the cosmic star formation rate (SFR) density $(z \sim 2)$, the majority of the star formation in the universe occurred behind dust (e.g., Madau \& Dickinson 2014). This has made it difficult to obtain a complete picture of galaxy evolution, particularly for the most actively star-forming population, which can be rendered faint or even invisible in the dust-sensitive restframe optical/UV imaging (e.g., Walter et al. 2012). In these galaxies, the majority of the rest-frame optical/UV light is reradiated in the far-infrared (FIR), resulting in large submillimeter flux densities for the high-redshift sources. Although such "submillimeter-selected galaxies" (SMGs; e.g., Blain et al. 2002; Casey et al. 2014) have been known about for over $20 \mathrm{yr}$ - and although they have been shown to contribute significantly to the cosmic SFR density (e.g., Swinbank et al. 2014)—-there is still considerable uncertainty over their detailed physical properties and overall nature.

The recent advent of the Atacama Large Millimeter Array (ALMA) is providing unique insights into high-redshift dusty star formation. In particular, the combination of ALMA's unprecedented sensitivity and resolution has allowed for spatially resolved (i.e., subgalactic) studies of the rest-frame 
FIR emission in the SMG population (e.g., Ikarashi et al. 2015; Simpson et al. 2015, 2017; Hodge et al. 2016; Chen et al. 2017; Calistro Rivera et al. 2018; Fujimoto et al. 2018), sometimes at even higher resolution than is possible in the optical $(\sim 0$ !' 03 ; e.g., Iono et al. 2016; Oteo et al. 2017; Gullberg et al. 2018). While there is still debate over where SMGs lie relative to the SFR-mass trend (e.g., da Cunha et al. 2015; Koprowski et al. 2016; Danielson et al. 2017; Elbaz et al. 2018), one thing that is becoming clear in all of these studies is that the distribution of dusty star formation (traced by the rest-frame FIR emission) is relatively compact $(\sim 3 \times$ smaller) compared to the rest-frame optical/UV emission visible with the Hubble Space Telescope (HST; e.g., Chen et al. 2015; Simpson et al. 2015; Calistro Rivera et al. 2018), and that it is disk-like on galaxy-wide scales (Sérsic index $n \sim 1$; e.g., Hodge et al. 2016).

There have been varying reports on whether the rest-frame FIR emission traced by ALMA submillimeter continuum observations shows evidence for structure on subgalactic scales. While some studies report evidence that a fraction of the submillimeter emission from some SMGs breaks up into "clumps" on subkiloparsec or even kiloparsec scales (e.g., Iono et al. 2016; Oteo et al. 2017), other studies find that the bulk of the observed emission is consistent with smooth disk emission given the signal-to-noise ratio (S/N; e.g., Hodge et al. 2016; Gullberg et al. 2018). Clumpy emission has been claimed previously on these scales based on observations of kiloparsecscale UV clumps in high-redshift galaxies (e.g., Dekel et al. 2009; Förster Schreiber et al. 2011; Guo et al. 2012, 2015), although there is little evidence that these represent true structures in the molecular gas or dust in these galaxies.

If the intense starbursts $\left(\sim 100->1000 M_{\odot} \mathrm{yr}^{-1}\right)$ observed in SMGs are triggered by galaxy interactions/mergers, as is commonly believed, then we might also expect to see morphological evidence of these interactions/mergers. In particular, it has long been known from early numerical work (e.g., Noguchi 1987) that tidal disturbances can induce the formation of nonaxisymmetric features such as galactic bars and spiral arms. Simulations suggest that spirals of the $m=2$ variety (i.e., double-armed) are actually difficult to produce except through tidal interactions/bars (Kormendy \& Norman 1979; Bottema 2003), with the most prominent grand-design spiral arms appearing in interacting galaxies such as M51. While the efficiency of their formation depends on the exact details of the orbital path and mass ratio (e.g., Athanassoula 2003; Lang et al. 2014; Kyziropoulos et al. 2016; Gajda et al. 2017; Pettitt \& Wadsley 2018), these nonaxisymmetric features can have significant consequences for the galactic dynamics. Specifically, they can interact with galactic material and cause resonances, including the corotation and inner and outer Lindblad resonances (OLRs; Sellwood \& Wilkinson 1993). Gas accumulates at these resonances and produces star-forming rings (e.g., Schwarz 1981; Buta 1986; Buta \& Combes 1996; Rautiainen \& Salo 2000). More critically, nonaxisymmetric features such as bars can also efficiently redistribute the angular momentum of the baryonic and dark matter components of disk galaxies (e.g., Weinberg 1985; Athanassoula \& Misiriotis 2002; Marinova \& Jogee 2007), triggering gas inflow and nuclear starbursts and thus driving spheroid growth.

The physical processes that accompany the intense bursts of star formation seen in systems such as SMGs and ultraluminous infrared galaxies (ULIRGs) are also thought to create feedback on the star-forming gas, potentially even slowing or halting further gravitational collapse in a self-regulating process. In particular, radiation pressure from massive stars on dust (which is coupled to the gas through collisions and magnetic fields) may play an important role in regulating star formation in the optically thick centers of starbursts like local ULIRGs (Scoville 2003; Murray et al. 2005; Thompson et al. 2005; Andrews \& Thompson 2011), where almost all of the momentum from the starlight is efficiently transferred to the gas. Indeed, Thompson et al. (2005) showed that radiation pressure could make up the majority of the vertical pressure support in so-called "Eddington-limited" dense starbursts.

While the latest ALMA results show that most SMGs are not approaching the Eddington limit for star formation on galaxywide scales (e.g., Simpson et al. 2015), this does not mean that the star formation is not limited by radiation pressure on more local (kiloparsec or subkiloparsec) scales, as has been observed in more compact local ULIRGs (e.g., Barcos-Muñoz et al. 2017) or even for giant molecular clouds in our own Milky Way (e.g., Murray \& Rahman 2010; Murray 2011). Similarly, while the bulk of the submillimeter emission in SMGs appears to be arising from a disk-like distribution on $\gtrsim$ kiloparsec scales, this does not mean that these dust and gas disks are featureless. In answering these open issues, obtaining higher angular resolution does not necessarily help unless one has correspondingly better surface brightness sensitivity to map the significance of beam-sized features with adequate $\mathrm{S} / \mathrm{N}$ (e.g., Hodge et al. 2016).

In this work, we present high-resolution $(\sim 0$."07), highfidelity ALMA imaging of the submillimeter emission (restframe FIR emission) from six SMGs at redshifts $1.5<z<4.9$ from the ALMA follow-up of the LABOCA ECDFS submillimeter survey (ALESS; Hodge et al. 2013), allowing us to study the morphology and intensity of their dusty star formation on $\sim 500 \mathrm{pc}$ scales. We present the details of the observations and data reduction in Section 2. The results are presented in Section 3, including a comparison with $H S T$ imaging (Section 3.1), an analysis of the subkiloparsec structure (Section 3.2), the presentation of SFR density maps (Section 3.3), and a comparison to the SFR-mass trend (Section 3.4). Section 4 presents a discussion of these results, followed by a summary of the conclusions in Section 5 . Throughout this work, we assume a standard $\Lambda$ CDM cosmology with $H_{0}=67.8 \mathrm{~km} \mathrm{~s}^{-1} \mathrm{Mpc}^{-1}, \Omega_{\Lambda}=0.692$, and $\Omega_{M}=0.308$ (Planck Collaboration et al. 2016).

\section{Observations and Data Reduction}

\subsection{ALMA Sample Selection and Observations}

The ALMA observations presented here were taken in six observing blocks from 2017 July 28 to August 27 as part of project \#2016.1.00048.S. In order to maximize $\mathrm{S} / \mathrm{N}$ for the high-resolution observations requested, the six SMGs were selected as the submillimeter-brightest sources from the 16 ALESS SMGs with previous high-resolution (0". 16) $870 \mu \mathrm{m}$ ALMA imaging from Hodge et al. (2016), which were themselves chosen as the submillimeter-brightest sources with (randomly targeted) HST coverage. All of the sources have existing HST data from CANDELS or our own program (Chen et al. 2015). No preselection was made on morphology/scale of the emission in the previous ALMA or HST imaging so as to avoid biasing the results. 
Table 1

Galaxy Properties

\begin{tabular}{|c|c|c|c|c|c|}
\hline Source ID ${ }^{\mathrm{a}}$ & $z^{\mathrm{b}}$ & $z_{\text {source }}{ }^{b}$ & $\overline{\log \left(M_{*} / M_{\odot}\right)^{\mathrm{c}}}$ & $\log \left(\mathrm{SFR} / M_{\odot} \mathrm{yr}^{-1}\right)^{\mathrm{c}}$ & 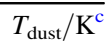 \\
\hline ALESS 3.1 & 3.374 & $\mathrm{CO}(4-3)$ & $11.30_{-0.24}^{+0.19}$ & $2.81_{-0.08}^{+0.07}$ & $36_{-2}^{+5}$ \\
\hline ALESS 9.1 & 4.867 & $\mathrm{CO}(5-4)$ & $11.89_{-0.12}^{+0.12}$ & $3.16_{-0.08}^{+0.07}$ & $51_{-4}^{+5}$ \\
\hline ALESS 15.1 & 2.67 & $z_{\text {phot }}$ & $11.76_{-0.26}^{+0.21}$ & $2.44_{-0.26}^{+0.15}$ & $33_{-4}^{+7}$ \\
\hline ALESS 17.1 & 1.539 & $\mathrm{H} \alpha, \mathrm{CO}(2-1)$ & $11.01_{-0.07}^{+0.08}$ & $2.29_{-0.03}^{+0.02}$ & $28_{-0}^{+6}$ \\
\hline ALESS 76.1 & 3.389 & [O III $]$ & $11.08_{-0.34}^{+0.29}$ & $2.56_{-0.12}^{+0.11}$ & $37_{-4}^{+10}$ \\
\hline ALESS 112.1 & 2.315 & $\operatorname{Ly} \alpha$ & $11.36_{-0.12}^{+0.09}$ & $2.40_{-0.08}^{+0.07}$ & $31_{-2}^{+5}$ \\
\hline
\end{tabular}

Notes.

${ }^{a}$ Source IDs are from Hodge et al. (2013).

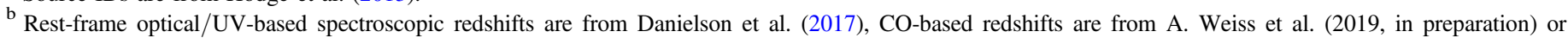
J. L. Wardlow et al. (2019, in preparation), and the photometric redshift was taken from da Cunha et al. (2015).

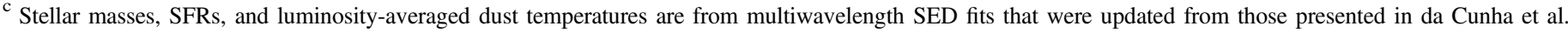

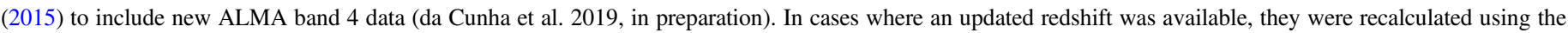
same method.

The observations were carried out in an extended configuration, with a maximum baseline of $3.7 \mathrm{~km}$. The average number of antennas present during the observations was 45 (with a range of 42-47). The 5th percentile of the baseline $u-v$ distances of the delivered data is $200 \mathrm{~m}$, giving a maximum recoverable scale (MRS) of 0!' 9 according to Equation (7.7) of the ALMA Cycle 4 Technical Handbook. This corresponds to a physical scale of $\sim 7.5 \mathrm{kpc}$ at a redshift of $z \sim 2.5$.

With the aim of quantifying the emission potentially resolved out by the requested extended-configuration observations, we utilized a spectral setup identical to the original Cycle 0 ALESS observations of these galaxies (Hodge et al. 2013), as well as the subsequent 0 ". 16 observations by Hodge et al. (2016). This setup is centered at $344 \mathrm{GHz}(870 \mu \mathrm{m})$ with $4 \times 128$ dual-polarization channels covering the $8 \mathrm{GHz}$ bandwidth. We utilized ALMA's Band 7 in Time Division Mode (TDM). At the central frequency, the primary beam is 17!"3 (FWHM). The total on-source time for each of the science targets was approximately 50 minutes, and we requested standard calibration. The median precipitable water vapor at zenith ranged from 0.4 to $1.0 \mathrm{~mm}$ across the six data sets, with an average value of $0.5 \mathrm{~mm}$.

Due to the selection criteria, the targets of this paper are some of the submillimeter-brightest sources of the ALESS SMG sample as a whole (Table 2; Hodge et al. 2013). They have redshifts that range from $\sim 1.5$ to 4.9 (Table 1), including five derived from optical and submillimeter spectroscopy (Danielson et al. 2017; A. Weiss et al. 2019, in preparation) and one from photometry (da Cunha et al. 2015). Their median redshift $(z=3.0 \pm 0.5)$ is consistent with the full ALESS sample $(z=2.7 \pm 0.1$; da Cunha et al. 2015). Their stellar masses, SFRs, and dust temperatures were derived from multiwavelength spectral energy distribution (SED) fits, which were updated from those presented in da Cunha et al. (2015) to include new ALMA Band 4 data (E. da Cunha et al. 2019, in preparation). Their median SFR $\left(\sim 300 M_{\odot} \mathrm{yr}^{-1}\right)$ is consistent with the ALESS sample as a whole (Swinbank et al. 2014; da Cunha et al. 2015), while their median dust temperature $(34 \pm 3 \mathrm{~K})$ is marginally cooler than the full sample as analyzed by da Cunha et al. (2015). Their median stellar mass $\left(\sim 2 \times 10^{11} M_{\odot}\right)$ is also larger than the median of the full sample $\left(\sim 8 \times 10^{10} M_{\odot}\right.$; Simpson et al. 2014), indicating that we may be probing the high-mass end of the population. One of the six sources is associated with an X-ray source and is classified as an active galactic nucleus (AGN; ALESS 17.1, $L_{0.5-8 \mathrm{keV} \text {,corr }}=1.2 \times 10^{43} \mathrm{ergs} \mathrm{s}^{-1}$; Wang et al. 2013).

\subsection{ALMA Data Reduction and Imaging}

The ALMA data were reduced and imaged using the Common Astronomy Software Application ${ }^{22}$ (CASA) version 4.7. Inspection of the pipeline-calibrated data tables revealed data of high quality, and the $u-v$ data were therefore used without further modification to the calibration scheme or flagging.

Prior to imaging, the data were combined with the lowerresolution $(\sim 0$ !' 16$)$, lower-sensitivity data previously obtained for these sources at the same frequency and presented in Hodge et al. (2016). Due to the lower sensitivity of the previous data, as well as the large MRS already achieved by the new data (Section 2.1), this made very little difference to the resulting image quality.

Imaging of the combined data was done using CASA's CLEAN task and multiscale CLEAN, a scale-sensitive deconvolution algorithm (Cornwell 2008). For this we employed a geometric progression of scales, as recommended, and we found that the exact scales used did not affect the outcome. The use of multiscale CLEAN made little qualitative difference to the final images, in comparison to those imaged without multiscale CLEAN, but we found that the residual image products from the runs without multiscale CLEAN showed a significant plateau of positive uncleaned emission that was absent in the residual maps made with multiscale CLEAN. We therefore use the multiscale CLEAN results for the remainder of the analysis.

Cleaning was done interactively by defining tight clean boxes around the sources and cleaning down to $1.5 \sigma$. Different weighting schemes were utilized on the $u-v$ data in order to produce images at different spatial resolutions and thus investigate the structure in the sources. As a point of reference, imaging the data with Briggs weighting (Briggs et al. 1999) and a robust parameter of $R=+0.5$-generally a good compromise between resolution and sensitivity-produced images with a synthesized beam size of 0 " $08 \times 0$ " 06 and a typical rms noise of $23 \mu \mathrm{Jy}$ beam $^{-1}$. With this array configuration and source $\mathrm{S} / \mathrm{N}$, the astrometric accuracy of

\footnotetext{
${ }^{22}$ http://casa.nrao.edu
} 
Table 2

$870 \mu \mathrm{m}$ Continuum Properties

\begin{tabular}{lccc}
\hline \hline Source ID & $\begin{array}{c}\text { Cycle 0 (1".5) } \\
(\mathrm{mJy})\end{array}$ & $\begin{array}{c}\text { This Work (0."3 } \\
\text { Taper) } \\
(\mathrm{mJy})\end{array}$ & $\begin{array}{c}\text { Recovered Fraction } \\
\ldots\end{array}$ \\
\hline ALESS 3.1 & $8.3 \pm 0.4$ & $8.7 \pm 0.2$ & $1.05 \pm 0.06$ \\
ALESS 9.1 & $8.8 \pm 0.5$ & $9.1 \pm 0.2$ & $1.03 \pm 0.06$ \\
ALESS 15.1 & $9.0 \pm 0.4$ & $9.6 \pm 0.2$ & $1.06 \pm 0.05$ \\
ALESS 17.1 & $8.4 \pm 0.5$ & $8.8 \pm 0.2$ & $1.04 \pm 0.06$ \\
ALESS 76.1 & $6.4 \pm 0.6$ & $5.0 \pm 0.1$ & $0.78 \pm 0.07$ \\
ALESS 112.1 & $7.6 \pm 0.5$ & $6.1 \pm 0.2$ & $0.80 \pm 0.06$ \\
\hline
\end{tabular}

the ALMA data is likely limited by the phase variations over the array to a few milliarcseconds. ${ }^{23}$

The MRS of the newly delivered data (0."9; Section 2.1) is larger than the median major-axis FWHM size of the ALESS sources at this frequency (0" $42 \pm 0$ ". 04; Hodge et al. 2016), indicating that most of the flux density should be recovered. To test this, we $u$ - $v$ tapered the concatenated data to $0 . .3$, cleaned them interactively, and measured the integrated flux densities, as the sources are still resolved at this resolution. The results are shown in Table 2, along with the flux densities measured from the compact-configuration $(\sim 1$ !' 6$)$ Cycle 0 observations (Hodge et al. 2013). In general, we recover most of the flux density measured in the lower-resolution Cycle 0 observations, indicating that the sources are relatively compact. For two of the six sources, the current data may be missing 20\% of the total $870 \mu \mathrm{m}$ emission, indicating the presence of a low surface brightness and/or extended component to the emission not recoverable in the present data. We therefore report any fractional contributions from structures detected in this work using the total flux densities derived in the lower-resolution Cycle 0 observations.

\subsection{HST Imaging}

We include in our analysis HST imaging from the Cosmic Assembly Near-infrared Deep Extragalactic Legacy Survey (CANDELS; Grogin et al. 2011; Koekemoer et al. 2011) and our own HST program (Chen et al. 2015). As presented in Chen et al. (2015), the combined data set on all 60 ALESS SMGs covered by these programs has a median point-source sensitivity in the $H_{160}$ band of $\sim 27.8 \mathrm{mag}$, corresponding to a $1 \sigma$ depth of $\mu_{\mathrm{H}} \sim 26 \mathrm{mag} \operatorname{arcsec}^{-2}$. The astrometry was corrected on a field-by-field basis using Gaia DR1 observations (Gaia Collaboration et al. 2016a, 2016b). The newly derived solutions were within $\lesssim 0$ ". 1 in both R.A. and decl. from the astrometric solutions previously derived by Chen et al. (2015) from a comparison with the $3.6 \mu \mathrm{m}$ Spitzer imaging.

\section{Results}

Figure 1 shows the ALMA maps of our six targeted SMGs, each imaged at three different spatial resolutions. At the redshifts of our targets (Table 1), $870 \mu \mathrm{m}$ corresponds to a restframe wavelength of $\sim 250 \mu \mathrm{m}$ (ranging from 150 to $350 \mu \mathrm{m}$ ), and a beam size of 0.107 corresponds to a typical spatial resolution of $\sim 500 \mathrm{pc}$ (ranging from 450 to $600 \mathrm{pc}$ ). All six sources show clear structure on these scales. The significance (both statistically and physically) of these structures will be

\footnotetext{
23 ALMA Cycle 5 Technical Handbook, Chapter 10.6.6.
}

discussed in more detail in Section 3.2. Before we attempt to interpret the meaning of the observed ALMA structure, we first examine the global ALMA $+H S T$ morphologies of the sources.

\subsection{HST Comparison}

Figure 2 shows false-color images for our sources constructed using a combination of the ALMA and deep HST imaging in one or more bands (Section 2.3), where the latter allows us to probe the existing unobscured stellar distribution at lower $(0$ ". $15 / 1.2 \mathrm{kpc}$ at $z \sim 2.5)$ resolution. The first thing to notice is that there is no correlation between the potential clumpy structure revealed in the new ALMA imaging and the $H S T$ imaging for any of the galaxies. This is because the dust emission traced by ALMA is more compact than the HST sources, as noted in previous studies (Simpson et al. 2015; Hodge et al. 2016; Chen et al. 2017; Calistro Rivera et al. 2018). Nevertheless, a careful look at the position of the ALMA emission relative to the rest-frame optical/UV emission can provide insight on these sources. Detailed notes on individual sources follow below.

ALESS $3.1\left(z_{\mathrm{spec}}=3.374\right)$. The deep $H_{160}$-band imaging of this source was previously analyzed by Chen et al. (2015), who reported a single $H_{160}$-band component with an effective radius of $r_{e}=5.5 \pm 0.7 \mathrm{kpc}$ (the Sérsic index was fixed at $n=1.0$ owing to the low $\mathrm{S} / \mathrm{N}$ of the source). Comparing to our ALMA data, the centroid of this $H_{160}$-band "component" lies $\sim 0$ ". 5 $(\sim 3.5 \mathrm{kpc})$ south of the ALMA source, which itself appears embedded in more extended, low-S/N $H_{160}$-band emission. If the dusty starburst detected by ALMA is centered on the center of mass of this system, then this source may be experiencing significant differential obscuration.

ALESS $9.1\left(z_{\mathrm{spec}}=4.867\right)$. The HST images are blank at the position of the ALMA-detected emission. There is a possible faint detection in the $H_{160}$-band emission, but it is offset $\sim 0$ ". 8 south of the ALMA source. The $I_{814}$-band CANDELS image is marred by an artifact near the ALMA source position but is otherwise blank.

ALESS $15.1 \quad\left(z_{\text {phot }}=2.67\right)$. The source is undetected in the $I_{814}$ band and has an extended, clumpy morphology in the $H_{160}$-band imaging. Like ALESS 3.1, it is possible that the ALMA emission (which shows a distinct curvature over its $\sim 10 \mathrm{kpc}$ extent; see also Figure 1) is centered on a more extended system that is suffering from differential dust obscuration.

ALESS 17.1 $\left(z_{\text {spec }}=1.539\right)$. The false-color image for ALESS 17.1 shows that the bulk of the ALMA $870 \mu \mathrm{m}$ emission lies offset $(\sim 0$.! 75$)$ from a disk galaxy in the HST imaging (though we do detect some very faint $870 \mu \mathrm{m}$ emission near the optical galaxy's nucleus). The galaxy detected in ALMA emission is undetected in HST imaging. Recent SINFONI imaging of the field (PI Swinbank) reveals $\mathrm{H} \alpha$ emission from both the optically detected galaxy and the ALMA source, indicating that they lie at the same redshift and are therefore likely interacting. Interestingly, this system is also associated with an X-ray AGN (Wang et al. 2013). The position of the X-ray source is indicated in Figure 2 as that reported by Luo et al. (2017), with an additional astrometric correction for the median offset reported between that X-ray catalog and Gaia DR1.

ALESS $76.1\left(z_{\mathrm{spec}}=3.389\right)$. This source appears completely undetected in the HST imaging ( $I_{814}$ band). We note that longer-wavelength $\left(H_{160}\right.$-band) imaging is not available. 


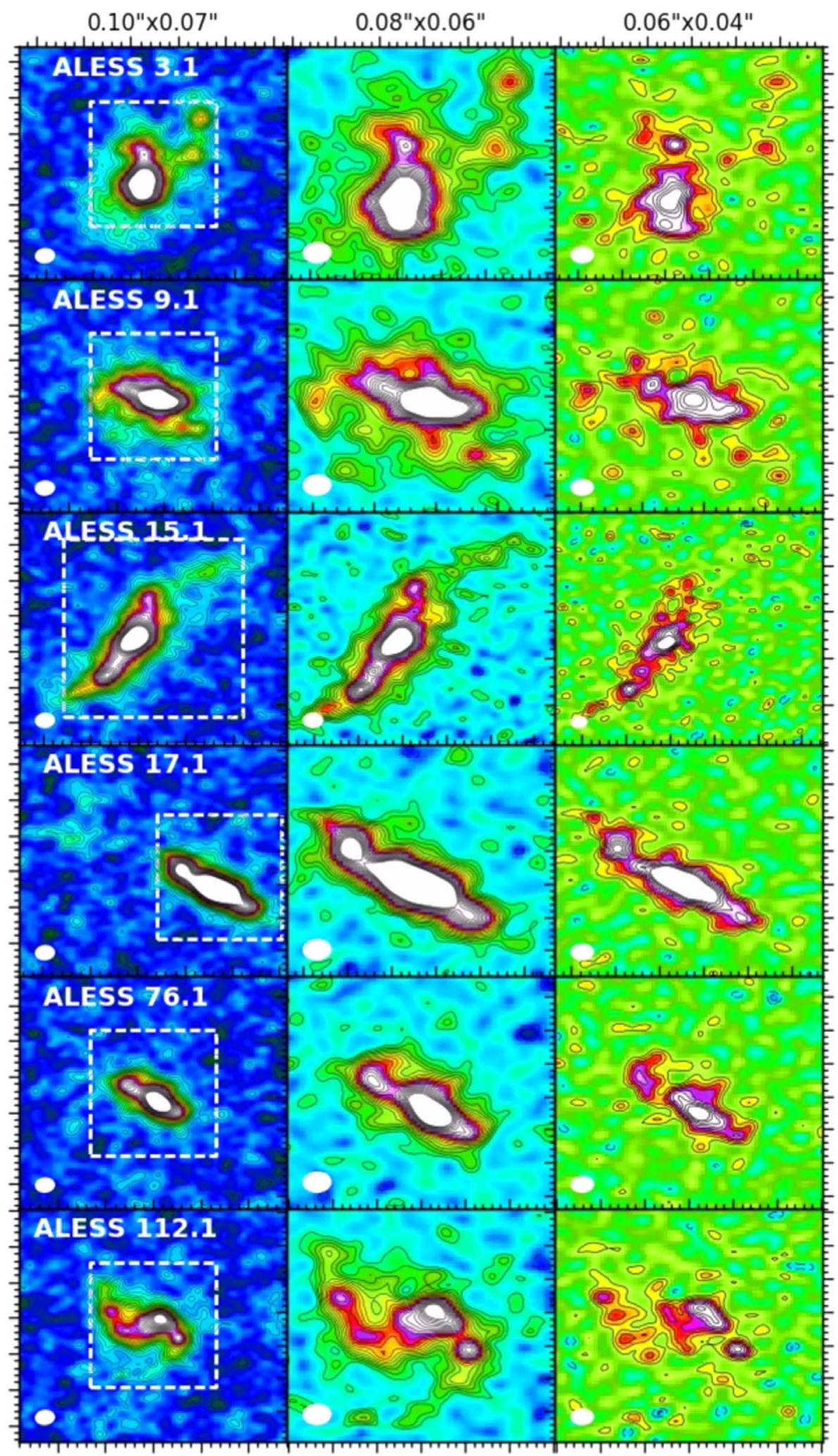

Figure 1. ALMA maps of the $870 \mu \mathrm{m}$ continuum emission from six SMGs imaged at three different resolutions (indicated above each column). Contours start at $\pm 2 \sigma$ and go in steps of $1 \sigma$, stopping at $30 \sigma$ (left), $20 \sigma$ (middle), and $10 \sigma$ (right) for clarity. These images reveal resolved structure on scales of $\sim 0$ !' 07 ( $\sim 500 \mathrm{pc}$ at $z \sim 2.5$ ), with large-scale structures suggestive of spiral arms and bars. Left column: 1." $3 \times 1$." 3 maps imaged with natural weighting, resulting in an rms of $\sigma \sim 20 \mu \mathrm{Jy}$ beam $^{-1}$ and a resolution of $0 ! ! 10 \times 0 !$. 07 . The dashed white box indicates the region shown in the two right columns and is $0 ! .7 \times 0 ! 7$ for all sources except ALESS 15.1, where a larger $1{ }^{\prime \prime} 0 \times 1{ }^{\prime \prime} 0$ region is shown. Middle column: zoomed-in maps of the region indicated in the left column, now imaged with Briggs weighting $(R=+0.5)$, resulting in an rms of $\sigma \sim 22 \mu \mathrm{Jy}$ beam ${ }^{-1}$ and a resolution of $0 . \prime 08 \times 0{ }^{\prime \prime} 06$. Right column: zoomed-in maps of the region indicated in the left column, now imaged with a different Briggs weighting $(R=-0.5)$, resulting in an rms of $\sigma \sim 42 \mu \mathrm{Jy}$ beam $^{-1}$ and a resolution of 0. " $06 \times 0$." 04 . 


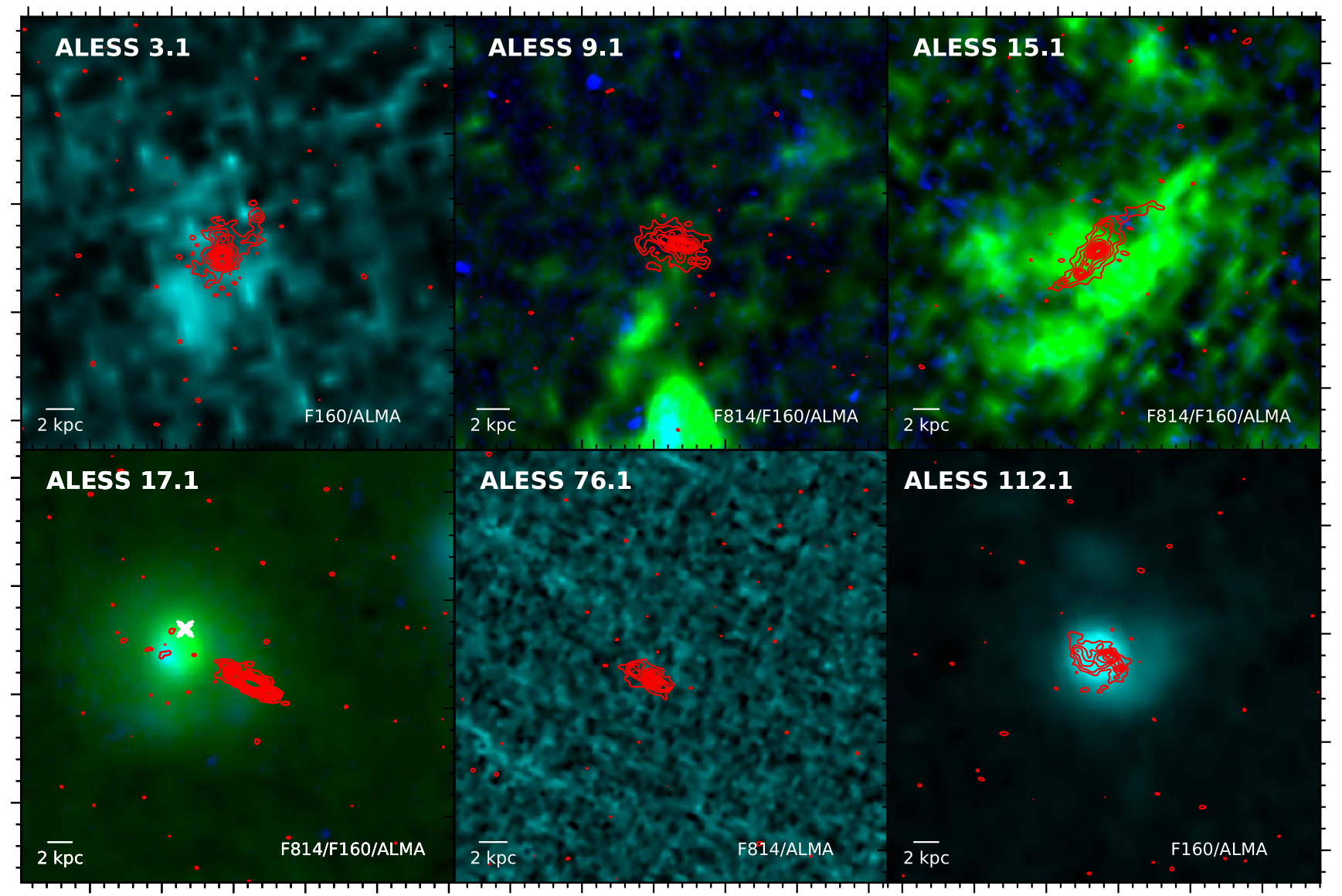

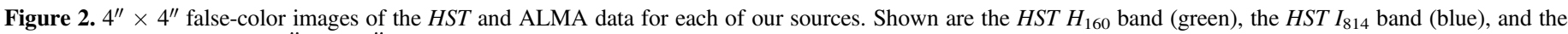

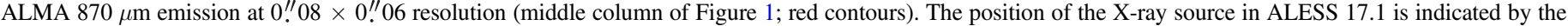

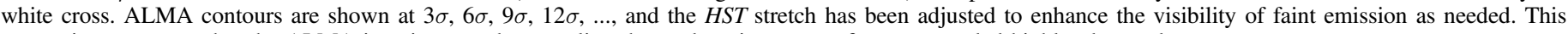
comparison suggests that the ALMA imaging may be revealing the starbursting cores of more extended highly obscured systems.

ALESS $112.1\left(z_{\mathrm{spec}}=2.315\right)$. The ALMA-detected $870 \mu \mathrm{m}$ continuum emission (which shows a prominent curvature over its $\sim 5 \mathrm{kpc}$ extent) appears by eye to be colocated with a bright counterpart in the $H S T H_{160}$-band imaging. The best-fit model to the $H_{160}$-band imaging has a Sérsic index of $n=3.4 \pm 1.3$ and an effective radius of $r_{e}=0 . " 59 \pm 0 . \prime 05$, corresponding to $4.9 \pm 0.4 \mathrm{kpc}$. This supports the conclusion that the high surface brightness $870 \mu \mathrm{m}$ (rest-frame FIR) emission is confined to the nucleus of a more extended stellar distribution.

In summary, despite the depth of the HST imaging (Section 2.3), the stellar emission from a number of the sources is extremely faint or invisible, making it challenging to characterize the rest-frame optical/UV morphologies of the systems. A superficial analysis shows that the majority of the HST-detected sources show offsets between the ALMA $870 \mu \mathrm{m}$ emission (tracing the rest-frame FIR) and the peak of the significantly detected emission in the deep HST imaging (tracing the unobscured rest UV/optical emission of these galaxies). These offsets are significant with respect to the relative astrometric accuracy of the two data sets (see Section 2.3). However, for at least half $(3 / 6)$ of the sources (and the majority detected in the HST imaging), extended HST emission surrounds the ALMA emission, indicating that the ALMA imaging may be revealing the heavily obscured starbursting cores of larger-scale systems. The comparison here highlights the need for sensitive high-resolution, near-/ mid-IR imaging of these dusty targets with a telescope such as the upcoming James Webb Space Telescope (JWST). We now turn to the statistical significance and possible interpretations of the new subkiloparsec dusty structure revealed by our ALMA data.

\subsection{Subkiloparsec FIR Structure}

The high-resolution ( $500 \mathrm{pc})$ images of our six SMGs presented in Figure 1 are generally dominated by an extended disk-like morphology - confirming the results of Hodge et al. (2016) based on shallower, lower-resolution data-but the new high-fidelity data presented here reveal new structures within these disks. We note that all visible structures were evident also in the dirty maps, indicating that they are not artifacts of the CLEANing process.

To assess the significance of the clumpy structure, we fit the galaxies with two-dimensional Sérsic profiles in GALFIT (Peng et al. 2002, 2010), masking residual pixels $>5 \sigma$ iteratively until the masks converged. This technique ensures that any real positive structure in the disks would not artificially boost the fits of the underlying smooth profiles, resulting in large negative troughs in the residual images. The resulting fits have half-light radii consistent with, and Sérsic indices that are on average slightly higher than, those derived without the masking procedure or from the lower-resolution data in Hodge et al. (2016) (with the notable exception of ALESS 112.1, which will be discussed further below). The results of this iterative procedure are shown in Figure 3, in which candidate structures 


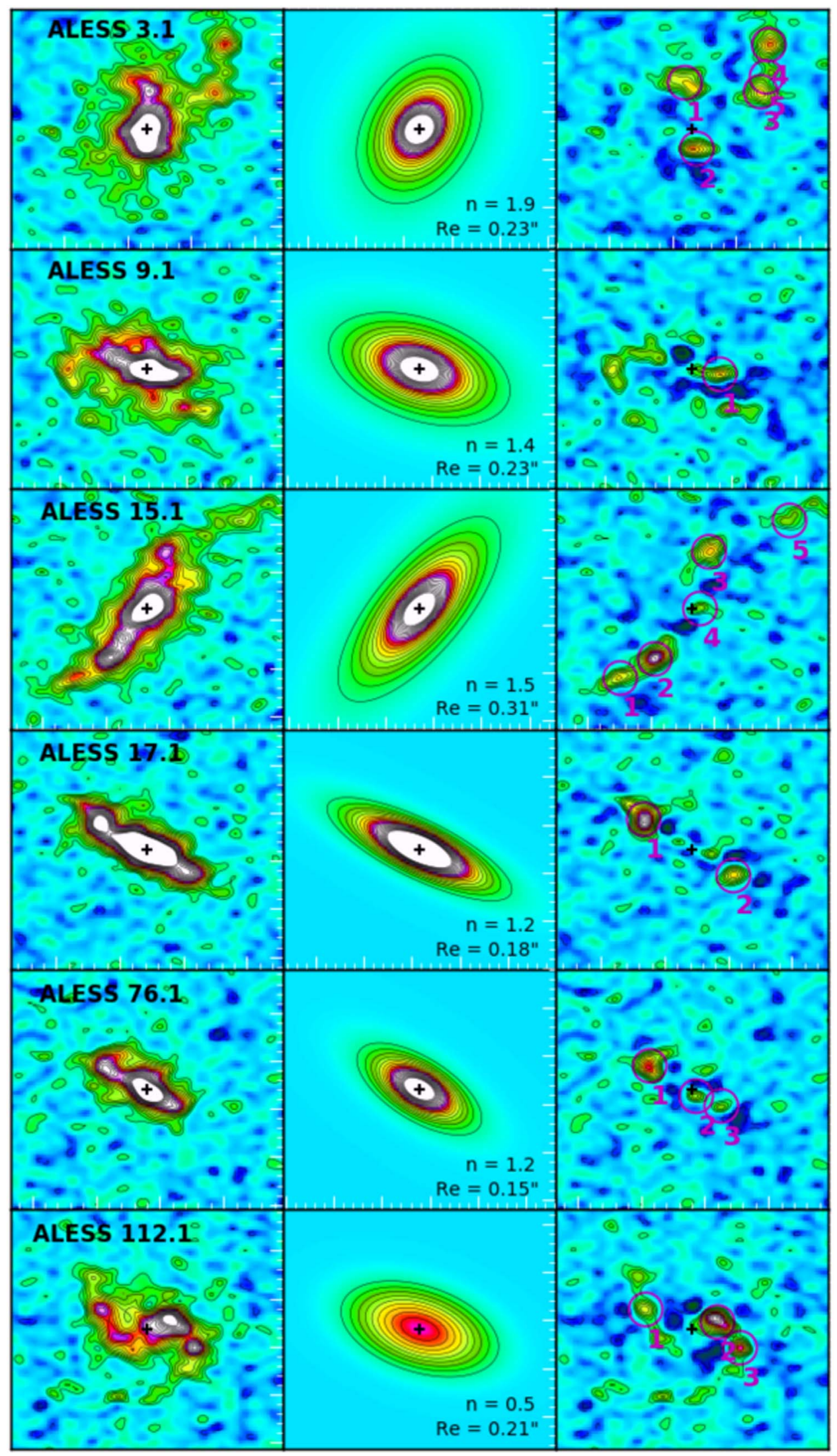

Figure 3. GALFIT modeling and substructure identification in our six galaxies as discussed in Section 3.2. Panels $\left(1{ }^{\prime \prime} 0 \times 1{ }^{\prime \prime} 0\right)$ show the observed maps with Briggs $(R=+0.5)$ weighting and $\sim 0$." $07 / 500 \mathrm{pc}$ resolution (left column), the best-fit Sérsic profile after masking residual pixels $>5 \sigma$ iteratively (middle column), and the residual maps resulting from the iterative masking (right column). The black plus sign marks the center of the model. Contours start at $\pm 2 \sigma$ and go in steps of $1 \sigma$, stopping at $20 \sigma$ for clarity. Structures more significant than the largest negative peak in each map are circled and labeled according to Table 3 . All six of the sources studied here show significantly detected complex dusty structure, including evidence for pairs of clump-like structures bracketing the elongated nuclear regions along the major axes of the most inclined sources. We discuss the possibility that we are observing inclined bar+ring morphologies in Section 4.3 . 
Table 3

Sérsic Profile Parameters and Properties of the Dusty Substructures

\begin{tabular}{|c|c|c|c|c|c|c|c|c|c|c|}
\hline Source & $\begin{array}{c}R_{e}{ }^{\mathrm{a}} \\
(\operatorname{arcsec})\end{array}$ & $n^{\mathrm{a}}$ & $b / a^{\mathrm{a}}$ & Structure $^{b}$ & $\mathrm{~S} / \mathrm{N}_{\mathrm{pk}}^{\mathrm{c}}$ & $\begin{array}{c}S_{\mathrm{pk}}^{\mathrm{c}} \\
\left(\mu \mathrm{Jy} \mathrm{beam}^{-1}\right)\end{array}$ & $\begin{array}{l}S_{\mathrm{int}^{\mathrm{c}}} \\
(\mu \mathrm{Jy})\end{array}$ & $\begin{array}{l}f_{\text {flux }}{ }^{\mathrm{d}} \\
(\%)\end{array}$ & $\begin{array}{l}b \mathrm{maj}^{\mathrm{e}} \\
(\mathrm{pc})\end{array}$ & $\begin{array}{c}b \min ^{\mathrm{e}} \\
(\mathrm{pc})\end{array}$ \\
\hline ALESS 3.1 & $0.23 \pm 0.01$ & $1.9 \pm 0.1$ & $0.68 \pm 0.02$ & $\begin{array}{l}1 \\
2 \\
3 \\
4 \\
5\end{array}$ & $\begin{array}{r}8.1 \\
10.0 \\
7.3 \\
8.8 \\
4.2\end{array}$ & $\begin{aligned} 180 & \pm 30 \\
220 & \pm 20 \\
160 & \pm 20 \\
190 & \pm 20 \\
90 & \pm 10\end{aligned}$ & $\begin{array}{l}530 \pm 100 \\
250 \pm 40 \\
390 \pm 80 \\
430 \pm 50 \\
220 \pm 40\end{array}$ & $\begin{aligned} 6 & \pm 1 \\
2.6 & \pm 0.3 \\
5 & \pm 1 \\
5.2 & \pm 0.7 \\
2.7 & \pm 0.5\end{aligned}$ & $\begin{aligned} & 1100 \pm 300 \\
& \ldots \\
& 800 \pm 200 \\
& 800 \pm 100 \\
& 1100 \pm 200\end{aligned}$ & $\begin{array}{c}500 \pm 200 \\
\ldots \\
500 \pm 300 \\
300 \pm 200 \\
100 \pm 200\end{array}$ \\
\hline ALESS 9.1 & $0.23 \pm 0.01$ & $1.4 \pm 0.1$ & $0.53 \pm 0.02$ & 1 & 8.6 & $190 \pm 20$ & $170 \pm 40$ & $2.2 \pm 0.3$ & $\cdots$ & $\cdots$ \\
\hline ALESS 15.1 & $0.31 \pm 0.01$ & $1.5 \pm 0.1$ & $0.37 \pm 0.02$ & $\begin{array}{l}1 \\
2 \\
3 \\
4 \\
5\end{array}$ & $\begin{array}{r}7.1 \\
13.0 \\
8.6 \\
5.2 \\
4.8\end{array}$ & $\begin{array}{l}160 \pm 10 \\
290 \pm 20 \\
190 \pm 20 \\
114 \pm 7 \\
105 \pm 7\end{array}$ & $\begin{aligned} 340 & \pm 40 \\
550 & \pm 60 \\
360 & \pm 50 \\
80 & \pm 10 \\
200 & \pm 20\end{aligned}$ & $\begin{array}{l}1.7 \pm 0.2 \\
6.1 \pm 0.7 \\
4.0 \pm 0.6 \\
1.3 \pm 0.1 \\
2.2 \pm 0.2\end{array}$ & $\begin{array}{c}\cdots \\
900 \pm 100 \\
800 \pm 200 \\
\cdots \\
900 \pm 100\end{array}$ & $\begin{array}{c}\cdots \\
200 \pm 100 \\
300 \pm 100 \\
\cdots \\
270 \pm 70\end{array}$ \\
\hline ALESS 17.1 & $0.18 \pm 0.01$ & $1.2 \pm 0.1$ & $0.26 \pm 0.01$ & $\begin{array}{l}1 \\
2\end{array}$ & $\begin{array}{r}15.5 \\
8.2\end{array}$ & $\begin{array}{l}340 \pm 40 \\
180 \pm 10\end{array}$ & $\begin{array}{l}660 \pm 100 \\
180 \pm 20\end{array}$ & $\begin{array}{c}8 \pm 1 \\
2.1 \pm 0.2\end{array}$ & $\begin{array}{c}800 \pm 100 \\
\ldots\end{array}$ & $\begin{array}{c}200 \pm 200 \\
\ldots\end{array}$ \\
\hline ALESS 76.1 & $0.15 \pm 0.01$ & $1.2 \pm 0.1$ & $0.40 \pm 0.02$ & $\begin{array}{l}1 \\
2 \\
3\end{array}$ & $\begin{array}{r}10.3 \\
6.4 \\
5.0\end{array}$ & $\begin{array}{l}230 \pm 30 \\
140 \pm 10 \\
110 \pm 20\end{array}$ & $\begin{aligned} 400 & \pm 90 \\
60 & \pm 20 \\
60 & \pm 20\end{aligned}$ & $\begin{array}{c}6 \pm 2 \\
2.2 \pm 0.3 \\
1.7 \pm 0.3\end{array}$ & $\begin{array}{c}600 \pm 200 \\
\ldots \\
\ldots\end{array}$ & $\begin{array}{c}200 \pm 300 \\
\ldots \\
\ldots\end{array}$ \\
\hline ALESS 112.1 & $0.21 \pm 0.01$ & $0.5 \pm 0.1$ & $0.52 \pm 0.04$ & $\begin{array}{l}1 \\
2 \\
3\end{array}$ & $\begin{array}{r}7.0 \\
16.0 \\
10.9\end{array}$ & $\begin{array}{l}150 \pm 10 \\
350 \pm 50 \\
240 \pm 20\end{array}$ & $\begin{array}{l}170 \pm 20 \\
540 \pm 100 \\
210 \pm 40\end{array}$ & $\begin{aligned} 2.0 & \pm 0.2 \\
7 & \pm 1 \\
3.2 & \pm 0.4\end{aligned}$ & $\begin{array}{c}\cdots \\
600 \pm 200 \\
\ldots\end{array}$ & $\begin{array}{c}\cdots \\
200 \pm 200 \\
\ldots\end{array}$ \\
\hline
\end{tabular}

Notes.

a Parameters from the best-fit Sérsic profile.

b Structure number as labeled in Figure 3.

${ }^{c}$ Peak S/N, peak flux density, and integrated flux density of the feature from a two-dimensional Gaussian fit in the image plane.

${ }^{\mathrm{d}}$ Fraction of the total flux density of the galaxy, measured from the compact-configuration (Cycle 0 ) values given in Table 2 .

e Deconvolved sizes. Blank entries indicate that the structure is unresolved at the current resolution $(0$ " $08 \times 0$.. 06$)$ and sensitivity.

are identified as structures more significant than the largest negative peak in each residual (i.e., Sérsic-subtracted) image. In general, between one and five residual structures are identified in each source at peak $\mathrm{S} / \mathrm{Ns}$ ranging from $\sim 4 \sigma$ to $15 \sigma$. Some of these structures lie near/within the nuclei and may be unresolved along one or both axes, indicating either real compact structure or a poor-fitting larger-scale profile (e.g., structure \#4 in ALESS 15.1), while others are clear "clumps" in the disk (e.g., structure \#1 in ALESS 17.1). Based on twodimensional Gaussian fits in the image plane, these structures individually make up a few percent $(\sim 1 \%-8 \%)$ of the total continuum emission from the galaxies, with a combined contribution of $\sim 2 \%-20 \%$ for a given galaxy. We estimate a median systematic error of $\sim 20 \%$ due to remaining structure in the residual maps. The deconvolved major axes of the structures range from $600 \mathrm{pc}$ to $1.1 \mathrm{kpc}$ for the roughly half that are resolved. Their properties are summarized in Table 3.

Even with these high-resolution, high-S/N data, the disk-like component still dominates the emission in these galaxies. The Sérsic indices we derive for the extended component from the iterative masking and fitting procedure are typically disklike $(\langle n\rangle=1.3 \pm 0.3)$, consistent with those derived from the lower-resolution (0". 16) data of a larger sample in Hodge et al. (2016) $(\langle n\rangle=0.9 \pm 0.2)$. One source (ALESS 112.1) has a very low $(n=0.5)$ Sérsic index. This source also has a large clump-like structure identified very near to the nucleus itself, indicating that a Sérsic profile may not be appropriate for the complex morphology seen here, which also shows a pronounced curvature.
Beyond the presence of these clumpy structures, their orientation may provide some clue as to their nature. In particular, in at least three of the sources (ALESS 15.1, 17.1, and 76.1), we see a significant clump-like structure on either end of an elongated nuclear region and oriented approximately along the major axis. We will discuss a possible interpretation for these features in Section 4.3.

\subsection{Star Formation Rate Surface Density Maps}

While the long-wavelength submillimeter emission in highredshift galaxies can be used to trace the total ISM mass via empirical calibrations (e.g., Scoville et al. 2014, 2016, 2017), it also correlates with the total SFR via the Kennicutt-Schmidt star formation law. For very dust-obscured galaxies like SMGs, which are difficult to observe in other commonly used resolved SFR tracers (e.g., $\mathrm{H} \alpha$ ), studies often rely on high-resolution submillimeter imaging to create maps of resolved SFR surface density ( $\Sigma_{\mathrm{SFR}}$; e.g., Hatsukade et al. 2015; Hodge et al. 2015; Cañameras et al. 2017; Chen et al. 2017). This is done by assuming that the variations in the observed submillimeter flux correlate with variations in the local SFR and scaling the total SFR by the observed-to-total ALMA $870 \mu \mathrm{m}$ flux density per beam across a source. The technique relies on having total (global) SFRs for each galaxy that are well determined through multiwavelength SED fitting. More critically, it effectively assumes that there are no variations in dust temperature $\left(T_{\mathrm{d}}\right)$ or emissivity index $(\beta)$ within the sources, which is unlikely to be correct. Nevertheless, it provides a first estimate of the distribution of $\Sigma_{\mathrm{SFR}}$ in these sources on $\sim 500 \mathrm{pc}$ scales. 

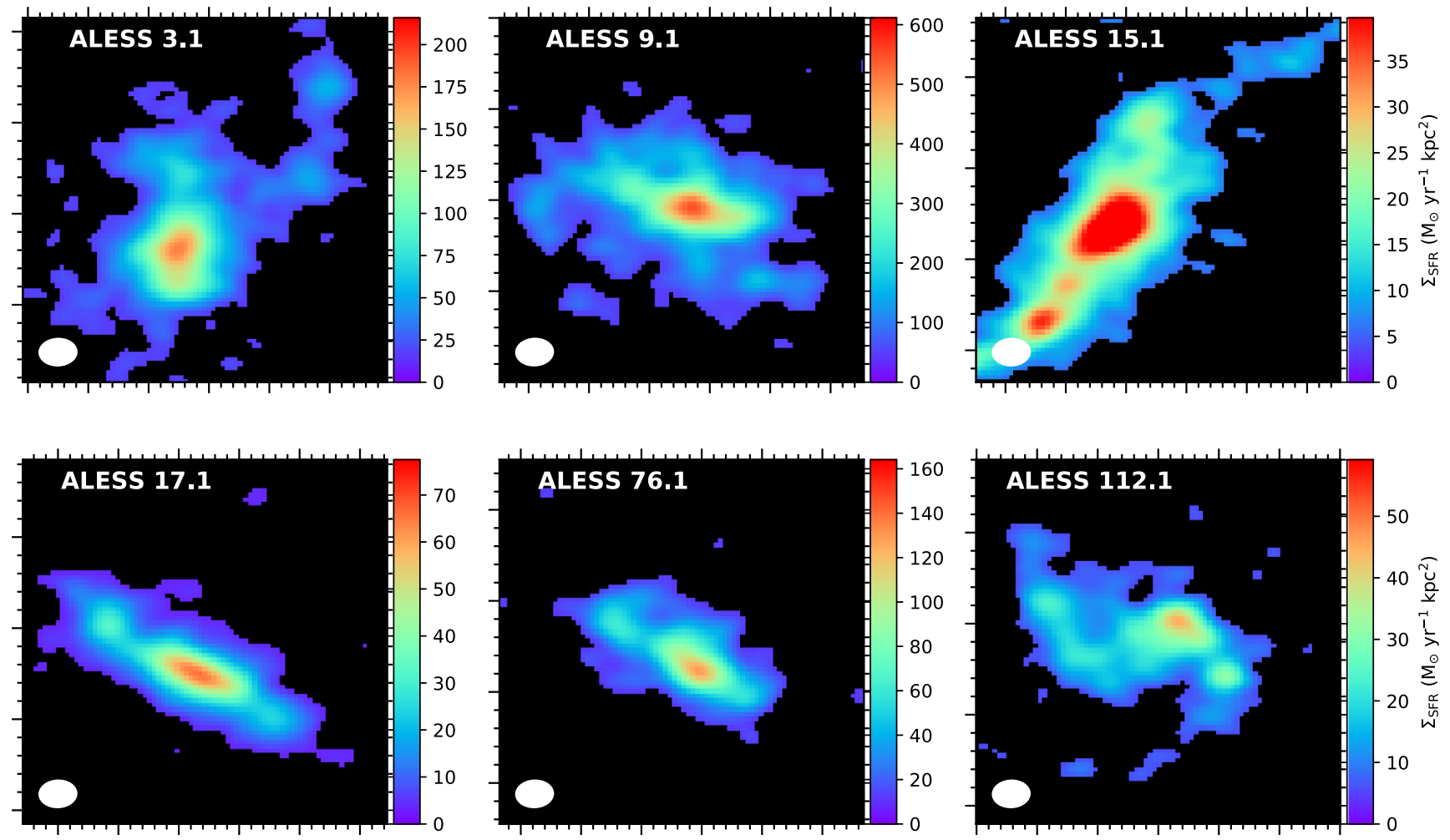

Figure 4. SFR surface density $\left(\Sigma_{\mathrm{SFR}}\right)$ maps at $\sim 0$ ! $07 / 500 \mathrm{pc}$ resolution (corresponding to the middle column of Figure 1 ), where emission below $3 \sigma$ has been masked. The beam is shown as the white ellipse in the lower left corner. By taking the global SFRs and dust temperatures derived for the galaxies through multiwavelength SED fitting (Table 1), we find that the range of $\Sigma_{\text {SFR }}$ probed varies between galaxies by over an order of magnitude. This is largely due to the similar $S_{870}$ values and sizes but very different (global) dust temperatures assumed for the galaxies.

The total far-infrared luminosities (and thus SFRs) for our galaxies are well constrained by the SEDs for the sources, which have been modified from those presented in da Cunha et al. (2015) to include updated redshift information and additional (unresolved) submillimeter observations in ALMA's Band 4 (E. da Cunha et al. 2019, in preparation). Following the above method, we created maps of SFR surface density $\left(\Sigma_{\mathrm{SFR}}\right)$ for our six sources $^{24}$ (Figure 4). We show the 0!"07-resolution maps in Figure 4. The peak values from maps at both resolutions, as well as the galaxy-averaged values calculated using the halflight radii as $0.5 \times \mathrm{SFR} /\left(\pi R_{e}^{2}\right)$, are listed in Table 4 .

The first thing to notice about Figure 4 is that the peak $\Sigma_{\mathrm{SFR}}$ on $\sim 500 \mathrm{pc}$ scales varies by over an order of magnitude between galaxies. As the peak $870 \mu \mathrm{m}$ flux densities only vary between galaxies by at most a factor of two and the physical scale of the emission is similar between galaxies, this is not solely a result of different observed flux density distributions. Rather, this large variation in peak $\Sigma_{\mathrm{SFR}}$ can be traced back to intrinsically different total SFRs (ranging from $\sim 150$ to $1500 M_{\odot} \mathrm{yr}^{-1}$ ), and ultimately to different physical conditions (dust luminosities and dust temperatures) in the sources (Table 1). These different dust temperatures/luminosities are constrained by the peak of the dust SED, which is typically reasonably well sampled in these sources: all six sources have five photometric data points between $\sim 200 \mu \mathrm{m}$ and $\sim 1.2 \mathrm{~mm}$ (observed frame), with only one source (ALESS 76.1) constrained by upper limits alone in the Herschel bands (Swinbank et al. 2014). We also note that this large range of

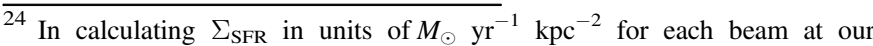
resolution, we note that beam area is defined as $\pi /(4 \times \ln (2)) \times b_{\text {maj }} \times b_{\text {min }}$.
}

Table 4

Inferred Star Formation Rate Densities

\begin{tabular}{lccc}
\hline \hline Source ID & $\begin{array}{c}\text { Mean } \Sigma_{\text {SFR }} \\
\left(M_{\odot} \mathrm{yr}^{-1}\right. \\
\left.\mathrm{kpc}^{-2}\right)\end{array}$ & $\begin{array}{c}\text { Peak } \Sigma_{\mathrm{SFR}} \\
\text { at } 0^{\prime \prime} 07\end{array}$ & $\begin{array}{c}\text { Peak } \Sigma_{\mathrm{SFR}} \\
\text { at } 0^{\prime \prime} 05\end{array}$ \\
\hline ALESS 3.1 & $33_{-15}^{+8}$ & $180_{-30}^{+31}$ & $\left.\mathrm{yr}^{-1} \mathrm{kpc}^{-2}\right)$ \\
ALESS 9.1 & $102_{-32}^{+27}$ & $547_{-93}^{+102}$ & $\left.\mathrm{yr}^{-1} \mathrm{kpc}^{-2}\right)$ \\
ALESS 15.1 & $7_{-3}^{+3}$ & $63_{-29}^{+26}$ & $572_{-108}^{+416}$ \\
ALESS 17.1 & $13_{-3}^{+3}$ & $66_{-6}^{+5}$ & $84_{-39}^{+35}$ \\
ALESS 76.1 & $44_{-26}^{+15}$ & $129_{-35}^{+39}$ & $77_{-8}^{+6}$ \\
ALESS 112.1 & $13_{-4}^{+3}$ & $45_{-9}^{+9}$ & $163_{-45}^{+51}$ \\
\hline
\end{tabular}

SFRs is not driven by our particular choice of SED-fitting code (MAGPHYS; da Cunha et al. 2015), as instead using simple modified blackbody fits with, e.g., the Kennicutt (1998) IRSFR relation (rescaled to the Chabrier 2003 initial mass function [IMF]) returns the same results (Swinbank et al. 2014). Physically, the measurement of a colder integrated dust temperature could indicate a larger contribution from dust heated by older stars (da Cunha et al. 2008), or it could indicate that the stellar radiation field seen by dust grains is not as intense. This is partly a selection effect, as the coldest sources are primarily at lower redshifts. Alternately, it could also be an artifact introduced in the SED modeling by assuming optically thin dust when it is indeed optically thick, depleting the emission at the shorter infrared wavelengths (e.g., Scoville 2013; Simpson et al. 2017).

An artifact of the difference in absolute scaling between galaxies is that the faintest $\Sigma_{\text {SFR }}$ we are sensitive to also varies 
between galaxies. For ALESS 9.1 (which has the highest peak $\Sigma_{\mathrm{SFR}}$ ), the $3 \sigma$ cutoff corresponds to $50 M_{\odot} \mathrm{yr}^{-1} \mathrm{kpc}^{-2}$. In ALESS 15.1, on the other hand, the $3 \sigma$ cutoff corresponds to $2.6 M_{\odot} \mathrm{yr}^{-1} \mathrm{kpc}^{-2}$. This limit is (again) affected by the assumption of a single (global) temperature over the sources.

Another assumption in the above analysis is that the rest-frame FIR emission is due to star formation rather than AGN activity. While this is generally thought to be true for the SMG population (e.g., Alexander et al. 2005; Laird et al. 2010), we note that one of our sources (ALESS 17.1; $L_{0.5-8 \mathrm{keV} \text {,corr }}=1.2 \times 10^{43} \mathrm{ergs} \mathrm{s}^{-1}$ ) was classified by Wang et al. (2013) as an AGN based on its low effective photon index $\left(\Gamma_{\text {eff }}<1\right)$, indicating a hard X-ray spectrum of an absorbed AGN. Due to its low $L_{0.5-8 \mathrm{keV} \text {,corr }} /$ $L_{\text {FIR }}$ ratio, however, Wang et al. (2013) concluded that it almost certainly had little to no AGN contribution in the FIR band. Indeed, it is interesting to note that the peak $\Sigma_{\mathrm{SFR}}$ of ALESS 17.1 $\left(\sim 75 M_{\odot} \mathrm{yr}^{-1} \mathrm{kpc}^{-2}\right.$ ) is actually on the lower side of the range for the sources studied in this work, perhaps indicating that the AGN is not even dominant on the scales $(\sim 500 \mathrm{pc})$ probed here.

\subsection{Relation to the SFR-Mass Trend}

There has been significant discussion in the recent literature about the relation of SMGs to the SFR-mass trend (e.g., Daddi et al. 2007; Noeske et al. 2007). In particular, some studies find that SMGs are (on average) offset above the SFR-mass trend in the "starburst regime" (e.g., Danielson et al. 2017), while others argue that the majority are consistent with the high-mass end of the relation (e.g., Koprowski et al. 2016). In their study of the full sample of ALESS SMGs, da Cunha et al. (2015) found that $\sim 50 \%$ of $z \sim 2$ SMGs are consistent with lying on the SFR-mass trend and that this fraction increases at higher redshift, where the trend evolves to higher values of SFR.

There are significant uncertainties involved in placing any one SMG on this trend, as systematic uncertainties on the stellar mass, SFR, and definition of the SFR-mass trend itself (e.g., Whitaker et al. 2012, 2014; Speagle et al. 2014; Tomczak et al. 2016) can easily shift the points by an order of magnitude along a given axis. In particular, there is considerable uncertainty in deriving robust stellar masses for these extremely dusty sources (e.g., Hainline et al. 2011; Michałowski et al. 2014; da Cunha et al. 2015) — a difficulty that is highlighted by the HST nondetections seen in Figure 2. For these sources, the stellar masses are constrained mainly through detections in the IRAC bands and may carry significant systematic uncertainties (Figure 5). In addition, there is also considerable uncertainty in the definition of the SFR-mass trend itself (e.g., Whitaker et al. 2012; Speagle et al. 2014). Nevertheless, it is interesting to consider where the galaxies targeted in this work fall with respect to the SFR-mass trend and the overall population of ALESS galaxies, particularly as they constitute some of the brightest submillimeter galaxies in the sample (Hodge et al. 2013) and yet have values of peak $\Sigma_{\text {SFR }}$ that vary by over an order of magnitude (Section 3.3). In Figure 5, we show the positions of the galaxies studied in this work in relation to the properties of the full ALESS sample as derived in da Cunha et al. (2015). All six of our galaxies are consistent with the SFR-mass trend for massive galaxies at their redshifts, and thus we find no immediate evidence for a correlation between position with respect to the SFR-mass trend and $\Sigma_{\mathrm{SFR}}$.

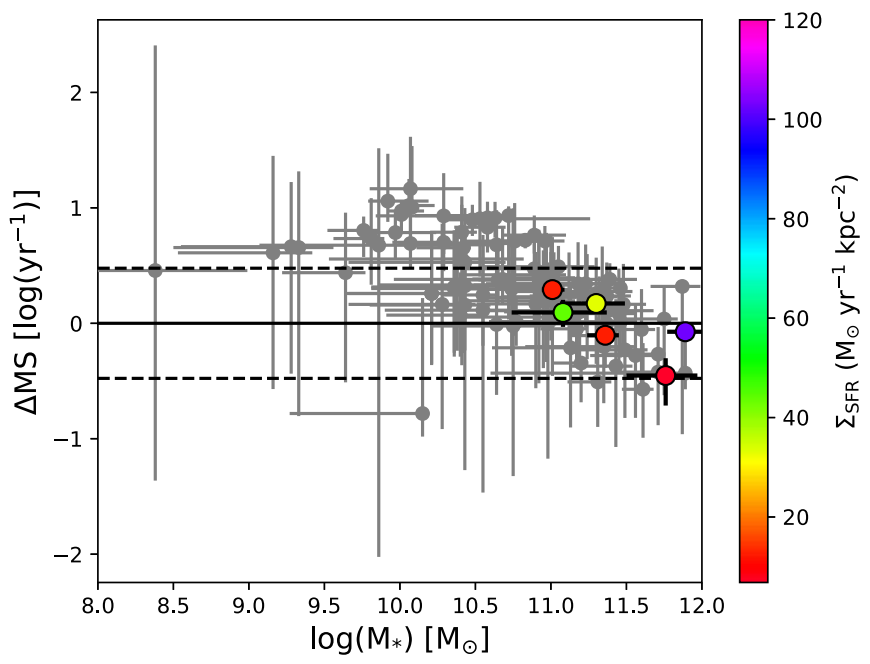

Figure 5. Distance from the star-forming SFR-mass trend $(\Delta \mathrm{MS}=$ $\mathrm{SFR} / \mathrm{SFR}_{\mathrm{MS}}$ ) vs. stellar mass for the galaxies studied in this work, where the data points are color-coded by galaxy-averaged SFR surface density. The gray points show the full ALESS SMG sample from da Cunha et al. (2015). As in da Cunha et al. (2015), the definition of the SFR-mass trend (solid line) is from Speagle et al. (2014), and the dashed lines indicate a factor of three above/below this relation. The error bars on the full ALESS sample are larger, as they include a marginalization over the redshift, which was a fitted parameter in da Cunha et al. (2015). Keeping in mind the considerable uncertainties in the creation of such a plot, we see that the six galaxies studied in this work are consistent with the SFR-mass trend for massive galaxies at their redshifts, and there is no correlation with total $\Sigma_{\mathrm{SFR}}$ within the sample.

\section{Discussion}

\subsection{The Intensity of the Star Formation}

Locally, radiation pressure on dust is thought to play an important role in regulating the star formation in the dense, optically thick centers of ULIRGs (e.g., Scoville 2003; Thompson et al. 2005). Recent ALMA work on SMG sizes has already demonstrated that they lie well below this "Eddington limit" on galaxy-wide scales (e.g., Simpson et al. 2015). We now investigate whether the SMGs also lie below the Eddington limit on the small physical scales probed here. To do this, we note that the theoretical value of the Eddington limit depends on the assumed physical conditions of the source (s). In particular, one important consideration is whether the galaxies are assumed to be optically thick to the reradiated FIR emission (i.e., $\tau_{\text {FIR }} \gtrsim 1$ ) in the dense centers of the starbursts. We can estimate whether this is true for our sources by solving the equation

$$
I_{\nu, \mathrm{obs}}=B_{\nu, \mathrm{rest}}\left(1-e^{\left.-\tau_{\nu, \mathrm{rest}}\right)},\right.
$$

where $\tau_{\nu}$ is the optical depth at frequency $\nu, B_{\nu, \text { rest }}=B_{\nu, \text { rest }}(T)$ is the Planck function, and $I_{\nu, \mathrm{obs}}$ is the specific intensity. The specific intensity can be calculated from the observations as

$$
I_{\nu, \mathrm{obs}}=0.5 S_{\nu, \mathrm{obs}}(1+z)^{3} / \Omega_{\nu, \mathrm{obs}},
$$

where $S_{\nu, \mathrm{obs}}$ is the observed (peak) flux density at our highest resolution, $z$ is the redshift, and $\Omega_{\nu, \mathrm{obs}}$ is the synthesized beam solid angle defined as $\pi /(4 \times \ln (2)) \times b_{\text {maj }} \times b_{\text {min }}$. Here we approximate the temperature $T$ required by the Planck function as the (global) dust temperature measured from the SEDs by MAGPHYS (Table 1), but we note that a more robust calculation of the true optical depths would require multifrequency, resolved data in the rest-frame far-infrared to break the degeneracy between dust 


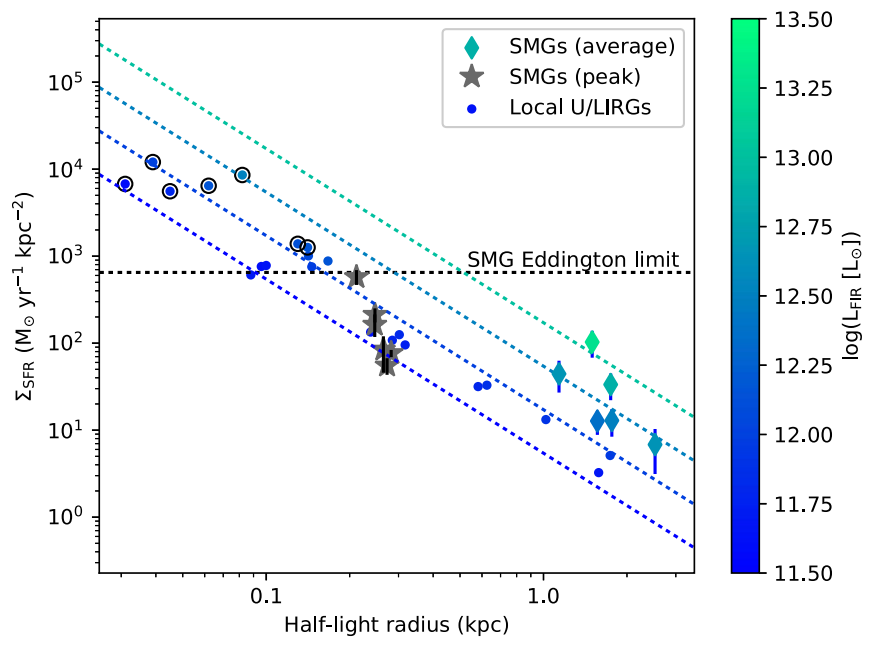

Figure 6. SFR surface density $\left(\Sigma_{\mathrm{SFR}}\right)$ vs. half-light radius for local U/LIRGs and the SMGs studied in this work. The local U/LIRGs come from BarcosMuñoz et al. (2017), where the $\Sigma_{\text {SFR }}$ values are galaxy averaged (rescaled to the Kennicutt 1998 IR-SFR conversion with the Chabrier 2003 IMF) and the half-light radii are the equivalent circular radii of the sources as observed at $33 \mathrm{GHz}$. For the SMGs in this work, both the galaxy-averaged and peak $\Sigma_{\mathrm{SFR}}$ values are shown, where the latter are calculated at our highest resolution (equivalent to half-light radii of $\sim 250 \mathrm{pc}$, with slight variations due to redshift). Both the local U/LIRGs and galaxy-averaged SMG points are color-coded by total FIR luminosity of the galaxy. Dashed diagonal lines indicate lines of constant FIR luminosity assuming the Kennicutt (1998) SFR $_{\text {IR }}$ calibration (rescaled to the Chabrier $2003 \mathrm{IMF}$ ). The horizontal dashed line indicates the estimated Eddington-limited SFR density for these SMGs assuming the optically thick limit in a warm starburst (Section 4.1). While Barcos-Muñoz et al. (2017) find that seven sources have IR surface brightnesses that exceed the characteristic limit for radiation pressure on dust for optically thick U/LIRGs (circled points; see Section 4.1), none of the SMGs exceed the Eddington limit on the resolved scales probed here.

temperature and optical depth (e.g., Simpson et al. 2017), as well as to map the temperature gradient within the sources. Nevertheless, solving for $\tau_{\nu \text {,rest }}$ using the above equations results in values of $\tau>1$ for rest-frame wavelengths of $\sim 150-350 \mu \mathrm{m}$, and the same is true if we assume that the central temperature is a factor of roughly a few higher. This result is consistent with that expected based on the typical gas surface densities in these sources (Simpson et al. 2015) for the "warm starburst" regime (Andrews \& Thompson 2011). We therefore assume that the dense centers of our sources are optically thick to FIR photons.

In this optically thick limit for warm starbursts, the Eddington flux is then shown by Andrews \& Thompson (2011) to be

$$
F_{\text {Edd }} \sim 10^{13} L_{\odot} \mathrm{kpc}^{-2} f_{\text {gas }}^{-1 / 2} f_{\mathrm{dg}, 150}^{-1},
$$

where $f_{\text {gas }}$ is the gas mass fraction. Noting that most of the bolometric luminosity from SMGs is emitted in the IR, and using the IR-based SFR calibration of Kennicutt (1998) (rescaled to a Chabrier 2003 IMF), we convert this to an Eddington-limited SFR density of

$$
\left(\Sigma_{\mathrm{SFR}}\right)_{\mathrm{Edd}} \sim 7.2 M_{\odot} \mathrm{yr}^{-1} \mathrm{kpc}^{-2} f_{\mathrm{gas}}^{-1 / 2} f_{\mathrm{dg}}^{-1}
$$

where we note that $f_{\mathrm{dg}, 150}=f_{\mathrm{dg}} \times 150$. Assuming the same dust-to-gas ratio as above (1/90) and adopting a gas fraction of unity as the most extreme scenario, we derive a lower limit on the Eddington-limited $\Sigma_{\mathrm{SFR}}$ of $\sim 650 M_{\odot} \mathrm{yr}^{-1} \mathrm{kpc}^{-2}$. As seen in Figure 6, none of the SMGs exceed this limit, even on the resolved scales probed here, and even in the individual clumplike structures (with the caveats stated above).

In Figure 6, we also compare our galaxy-averaged and peak $\Sigma_{\text {SFR }}$ values with the galaxy-averaged $\Sigma_{\text {SFR }}$ values derived for 22 local luminous and ultraluminous galaxies (U/LIRGs) from Barcos-Muñoz et al. (2017). These U/LIRGs were selected from the IRAS Revised Bright Galaxy Sample (RBGS; Sanders et al. 2003) as 22 of the most luminous sources in the northern sky, and they have a median FIR luminosity of $\sim 10^{11.8} L \odot$, corresponding to a median SFR of $\sim 80 M_{\odot} \mathrm{yr}^{-1}$. Their $\Sigma_{\mathrm{SFR}}$ values were calculated using IR-based SFRs and assuming that the $33 \mathrm{GHz}$ size reflects the distribution of the star formation. Their physical resolution ranged from 30 to $720 \mathrm{pc}$, and in some cases the sources were only marginally resolved.

We see in Figure 6 that the average $\Sigma_{\text {SFR }}$ values and halflight radii for the local U/LIRGs are fairly tightly correlated. The scatter in the correlation can be attributed to the range in total FIR luminosities for the U/LIRGs. The local U/LIRGs also span a much wider range in galaxy-averaged $\Sigma_{\text {SFR }}$ than the SMGs, which is largely due to the fact that the physical sizes of the U/LIRGs span $>1$ dex, whereas the SMG sizes are fairly homogeneous and much larger on average. ${ }^{25}$ For a given total source size, however, the SMGs can have galaxy-averaged $\Sigma_{\text {SFR }}$ values of up to an order of magnitude higher than U/LIRGs. This can be attributed to the larger total FIR luminosities of the SMGs. Looking at this from a different perspective, the results in Figure 6 suggest that SMGs are able to sustain a given galaxy-averaged $\Sigma_{\text {SFR }}$ over much larger physical extents.

However, we also see from Figure 6 that local U/LIRGs can achieve even higher $\Sigma_{\text {SFR }}$ values on smaller physical scales than are observable here. Indeed, Barcos-Muñoz et al. (2017) found that seven of their sources have IR surface brightnesses that exceed the characteristic limit of $\sim 10^{13} L_{\odot} \mathrm{kpc}^{-2}$ for radiation pressure on dust in optically thick U/LIRGs-even averaging over the sources-which Thompson et al. (2005) argue is consistent with that expected for a central gas fraction of $f_{\mathrm{g}} \sim 0.5$. For an even more conservative gas fraction of 0.3 , five of the U/LIRGs would still exceed the limit, indicating that they may be Eddington-limited starbursts. The apparent super-Eddington values could then be due to one of the assumptions in the calculation breaking down, such as the assumption of equilibrium in the system through the generation of a galactic wind. Interestingly, the peak $\Sigma_{\text {SFR }}$ values for the SMGs measured at the highest resolution (equivalent to halflight radii of $\sim 250 \mathrm{pc}$ ) are similar to those of U/LIRGs with that same total size. As the U/LIRGs only exceed the Eddington limit on smaller physical scales, this could indicate that even higher-resolution $(<500 \mathrm{pc}$ FWHM) observations would be necessary to observe super-Eddington star formation in SMGs.

One important caveat in the above analysis is the previously stated assumption of a single dust temperature across the sources. This assumption is unlikely to be true based on both detailed studies of resolved local galaxies (e.g., Engelbracht et al. 2010; Pohlen et al. 2010; Galametz et al. 2012) and radiative transfer modeling of the dust versus $\mathrm{CO}$ extents from a stacking analysis of the ALESS SMGs specifically, where the observations are well fit by radially decreasing temperature

\footnotetext{
25 Note that no preselection was made in our sample on morphology or scale of the submillimeter emission, as discussed in Section 2.1.
} 
gradients (Calistro Rivera et al. 2018). Assuming a dust temperature gradient that decreased with radius would change the distribution of the $\Sigma_{\mathrm{SFR}}$, causing it to peak at higher values in the center and decrease more rapidly in the outskirts. Determining the magnitude of this effect will require resolved, high-S/N multiband continuum mapping of these high-redshift sources to map their internal dust temperature gradients with ALMA.

\subsection{Dusty Substructure in SMGs}

The high-resolution, high-S/N ALMA $870 \mu \mathrm{m}$ imaging of SMGs presented in this work confirms the disk-like morphology of the dusty star formation in these galaxies (Hodge et al. 2016; Gullberg et al. 2018), which-although very compact relative to the $H S T$ imaging-is more extended than similarly luminous local galaxies in the FIR (e.g., Arp 220). If we interpret the structures we observe in our galaxies as starforming "clumps"- defined as discrete star-forming regions such as those claimed in rest-frame optical/UV imaging (e.g., Guo et al. 2012, 2015), near-infrared integral field spectroscopy (e.g., Förster Schreiber et al. 2011), and molecular gas imaging (e.g., Tacconi et al. 2010; Hodge et al. 2012) of high-redshift galaxies - this allows us to place some first constraints on the importance of these structures to the global star formation in these massive, dusty sources. We find that they each contain only a few percent of the emission in a given galaxy, with a combined contribution of $\sim 2 \%-20 \%$ and an additional systematic error of $20 \%$ (Section 3.2). Assuming a constant internal dust temperature (Section 3.3), this would imply that kiloparsec-scale clumps are not the dominant sites of star formation in these SMGs. If the clump-like structures we observe trace sites of young massive star formation, then the dust temperature in these regions may be higher than the quiescent regions (e.g., Galametz et al. 2012), implying that their fractional contribution to the global SFRs may be higher. We also note that the (luminosity-weighted) temperatures estimated by our global measurements are likely to be dominated by the star-forming regions seen in the continuum maps (e.g., Utomo et al. 2019).

For comparison, hydro-cosmological zoom simulations of giant clumps in $1<z<4$ disk galaxies have previously examined the contribution of both in situ (via violent disk instability) and ex situ (via minor mergers) clumps to the total SFRs, finding that a central "bulge clump" alone usually accounts for $23 \%$ (on average) of the total SFR (Mandelker et al. 2014). This is a larger contribution than we identified in any of our clump-like structures-regardless of position relative to the bulge-although we are implicitly assuming that such clumps would still be identifiable in our Sérsic fits to the continuum emission (as opposed to the molecular gas line emission in 3D, as done in the simulations). Only considering off-center clumps, Mandelker et al. (2014) find an average SFR fraction in clumps of $20 \%$ (range $5 \%-45 \%$ ) - somewhat higher than we estimate, though these clumps are distributed over larger areas and the galaxies themselves are generally less massive $\left((0.2-3) \times 10^{11} M_{\odot}\right)$ and less highly star-forming than the galaxies imaged here.

The clumpy structures that we do significantly detect have (deconvolved) sizes ranging from unresolved (at $500 \mathrm{pc}$ resolution) to $\sim 1 \mathrm{kpc}$. Following Gullberg et al. (2018) and estimating the gas surface density from the global $\Sigma_{\mathrm{SFR}}$, we find that obtaining a Jeans length comparable to the largest
Table 5

Constraints on Velocity Dispersions

\begin{tabular}{lcc}
\hline \hline Source ID & $\begin{array}{c}\text { Jeans Length Constraint } \\
\left(\mathrm{km} \mathrm{s}^{\mathrm{a}}\right)\end{array}$ & $\begin{array}{c}\text { Toomre Constraint }^{\mathrm{b}} \\
\left(\mathrm{km} \mathrm{s}^{-1}\right)\end{array}$ \\
\hline ALESS 3.1 & $>150$ & $\ldots$ \\
ALESS 9.1 & $\ldots$ & $\ldots$ \\
ALESS 15.1 & $>60$ & $<70$ \\
ALESS 17.1 & $>75$ & $<90$ \\
ALESS 76.1 & $>100$ & $<160$ \\
ALESS 112.1 & $>70$ & $\cdots$ \\
\hline
\end{tabular}

Notes.

${ }^{a}$ Minimum velocity dispersion necessary for the Jeans length to equal the major axis of the largest clump-like structure in that source (Table 3).

${ }^{\mathrm{b}}$ Maximum velocity dispersion allowable for a Toomre stability parameter $Q<1$ in the sources with potential bar-like features (Section 4.3).

clump-like feature observed in each source would require velocity dispersions of $\gtrsim 60-150 \mathrm{~km} \mathrm{~s}^{-1}$ (Table 5). While we do not have measured velocity dispersions for these sources specifically (though see Section 4.3), observations of other SMGs (lensed and unlensed) suggest values of $10-100 \mathrm{~km} \mathrm{~s}^{-1}$ (Hodge et al. 2012; De Breuck et al. 2014; Swinbank et al. 2015). Taking the value of $40 \mathrm{~km} \mathrm{~s}^{-1}$ measured previously for one source from the full ALESS sample (ALESS 73.1; De Breuck et al. 2014) gives Jeans lengths ranging from 50 to $400 \mathrm{pc}$. Therefore, while the above calculation assumes both velocity dispersion and gas surface density, it is possible that the largest clump-like structures that we observe either may be blends of smaller structures at the current beam size or may not be self-gravitating. We attempt to place further constraints on the velocity dispersion for a subset of sources below in Section 4.3.

\subsection{Evidence for Interaction, Bars, Rings, and Spiral Arms?}

A comparison between the high-resolution ALMA images and deep HST imaging provides further insight into these highly star-forming sources. In particular, for one source (ALESS 17.1), we see a submillimeter component that is significantly (spatially) offset from a separate optically detected disk galaxy. This offset is now confirmed as significant thanks to the resolution of ALMA and the astrometric solutions of Gaia. SINFONI spectroscopic imaging indicates that the submillimeter- and optically detected galaxies are at the same redshift (M. Swinbank 2019, personal communication), and thus we are likely witnessing an interaction-induced starburst in the ALMA source, which is itself undetected in the optical. Interestingly, this is also the only one of our sources associated with an X-ray AGN (Wang et al. 2013).

A more general observation from the HST comparison is that for at least half $(3 / 6)$ of the sources (and the majority detected in the HST imaging) a careful inspection suggests that the ALMA emission may be centered on disturbed and/or partially obscured optical disks. This then suggests that the ALMA imaging in these cases is tracing the dusty cores of more extended systems, and it also aids in the interpretation of the dusty substructure in the global picture.

In particular, in two of these sources (112.1/15.1), the morphology of the high-fidelity ALMA imaging shows a very clear curvature reminiscent of either spiral arms or the starforming knots in an interaction/merger such as the Antennae (Klaas et al. 2010). In this case, the scale of the emission is an 
important clue. From Figure 2, it is clear that the dusty structure revealed by the ALMA imaging is tracing the inner $\sim 5-10 \mathrm{kpc}$ of the systems and is thus inconsistent with larger-scale tidal features.

The curvature seen in the $870 \mu \mathrm{m}$ emission of ALESS 112.1 and 15.1 may then be revealing star-forming spiral structure, potentially induced by an interaction/tidal disturbance. While spiral arms are generally thought to emerge in galaxies only at redshifts of $z \lesssim 2$ (Elmegreen \& Elmegreen 2014), a handful of spiral galaxies have been claimed at higher redshift (a threearmed spiral at $z=2.18$ and a one-armed spiral at $z=2.54$; Law et al. 2012; Yuan et al. 2017). Of the spirals, grand-design $(m=2)$ spirals, such as our observations suggest, are thought to extend the furthest back in time, likely due to the ability of interactions to drive such spirals. Specifically, tidal interactions from prograde encounters are very effective at inducing the formation of spiral arms, particularly of the $m=2$ variety (Dobbs \& Baba 2014). The perturber should ideally be $1 / 10$ of the mass of the main galaxy to produce a clear grand-design spiral pattern (Oh et al. 2008). Their apparent rarity at high redshift is likely due not only to the fact that specific circumstances must be achieved to incite the spiral pattern in the first place (the galaxy must be massive enough to have stabilized the formation of an extended disk, and the disk must then be perturbed by a sufficiently massive companion with the correct orientation) but also to the fact that such interactiondriven spirals are generally short-lived (though this depends on the exact configuration and orbital parameters; Law et al. 2012). In that sense, and if this morphology is triggered by an interaction, it is perhaps not surprising that some of the ALESS SMGs show potential spiral structure, as they were selected through their bright submillimeter emission to be some of the most highly star-forming galaxies in the universe, ensuring that they are both massive and viewed close in time after the presumed interaction.

Alternately, the spiral structure visible in ALESS 112.1which is also the source with the lowest Sérsic index-may instead be due to a late-stage major merger viewed at a serendipitous angle. The maximum starburst (and heaviest dust obscuration) coincides with final coalescence in retrograderetrograde mergers, which also show appreciably larger internal dust extinction than prograde-prograde configurations (Bekki \& Shioya 2000). The fact that the strongly star-forming component is on average more compact than both the gas and existing stellar component in SMGs (Simpson et al. 2015; Chen et al. 2017; Calistro Rivera et al. 2018) would also be consistent with this picture (e.g., Bekki \& Shioya 2000).

In at least three of the sources (ALESS 15.1, 17.1, 76.1), we detect clump-like structures along the major axis of the ALMA emission, bracketing elongated nuclear emission. This could suggest that we are observing bars in the cores of these galaxies, where the aligned clump-like structures are either starforming gas complexes such as those frequently seen in local barred galaxies (e.g., NGC 1672), and which may be formed through orbit crowding in a bar-spiral transition zone (e.g., Kenney \& Lord 1991; Kenney et al. 1992), or that they are due to a star-forming ring that is visible as two clumps owing to the long path length where the line of sight is perpendicular. As the three sources with the strongest evidence for this morphology are also the most highly inclined sources based on
Table 6

Sources with Potential Bar+Ring Morphologies

\begin{tabular}{lccc}
\hline \hline Source ID & $\begin{array}{c}\text { "Bar" Radius } \\
(\mathrm{kpc})\end{array}$ & $\begin{array}{c}\text { "Ring" Radius } \\
(\mathrm{kpc})\end{array}$ & $\begin{array}{c}\text { Ratio }\left(\mathrm{OLR} / \mathrm{CR}^{\mathrm{c}}\right) \\
\ldots\end{array}$ \\
\hline ALESS 15.1 & $0.7 \pm 0.1$ & $1.9 \pm 0.1$ & $2.6 \pm 0.2$ \\
ALESS 17.1 & $1.0 \pm 0.1$ & $1.9 \pm 0.1$ & $1.5 \pm 0.2$ \\
ALESS 76.1 & $0.7 \pm 0.1$ & $1.3 \pm 0.1$ & $1.9 \pm 0.2$ \\
\hline
\end{tabular}

Notes.

a Defined as the HWHM of the central ("bar") component along the major axis from a two-dimensional Gaussian fit.

b Defined as the average distance from the source center to the "clumps" bracketing the central emission along the major axis.

c The ratio of the "ring" and "bar" sizes, taken here to indicate the ratio of the outer Lindblad resonance (OLR) and the corotation radius (CR). See Section 4.3 for further details.

the GALFIT modeling, this could be evidence for the latter (a bar and ring morphology).

Bars observed in the local universe in the near-infrared are usually well fit by Sérsic models with $n \sim 0.5-1$ (Weinzirl et al. 2009). It is difficult to separate the potential bars from the disks in a multicomponent Sérsic fit with the data presented here, so we can only say that these values are typically lower than what we measure for our galaxies on the whole. If we define the radius of the bar as the HWHM of the central component along the major axis from a two-dimensional Gaussian fit, then we find bar radii of $0.7-1 \mathrm{kpc}$ (Table 6). In Hoyle et al. (2011), a Galaxy Zoo project that measured bar lengths in $\sim 3000$ local Sloan Digital Sky Survey disk galaxies, they report bar radii of $\sim 1-10 \mathrm{kpc}$, which would put these high-redshift "bars" on the extreme short end. (The rings are correspondingly small for what you would then expect based on the bar resonances-see below). However, the same study finds that the shortest bars are found in the bluest (and thus presumably most star-forming) disk galaxies. This may be due to the gas content of the galaxies, as recent high-resolution hydrodynamic simulations of barred galaxies by Seo et al. (2019) find that higher gas fractions result in shorter bars, particularly in dynamically "cold" systems (based on the ratio of the radial and vertical velocity dispersions). In addition, based on the typical gas depletion timescales estimated for SMGs ( 100-200 Myr; Casey et al. 2014, and references therein), it is possible that we could be observing bars shortly after their formation, when they are expected to be shorter (e.g., Seo et al. 2019). The comparison is complicated by the fact that bar formation is a highly nonlinear process, especially in the presence of gas, and depends sensitively on the initial galaxy parameters (Seo et al. 2019). It is interesting to note that all of the bars formed in the Seo et al. (2019) hydrodynamic simulations eventually thicken and form bulges.

If our identification of these features is correct, and if we assume that the bar extends approximately to the corotation radius (CR) in these galaxies (Sanders \& Tubbs 1980; Tremaine \& Weinberg 1984; Buta 1986; Athanassoula 1992; Lindblad et al. 1996; Weiner et al. 2001; Pérez et al. 2012), then the extent of the bar can also give us the CR. In such a scenario, the rings form as a result of gas accumulation at the bar resonances, and the diameter of the rings gives the OLR. Taking these three galaxies (ALESS 15.1, 17.1, and 76.1), we define the radius of the bar as above and the radius of the "ring" 
as the average distance to the "clumps" from the source center, resulting in a median ratio of the two sizes (interpreted here as OLR/CR) of $1.9 \pm 0.3$. This ratio agrees with the OLR/CR ratio found for the local galaxy population ${ }^{26}$ (e.g., Kormendy 1979; Buta 1995; Laurikainen et al. 2013; Herrera-Endoqui et al. 2015), supporting this interpretation of these features. Notably, this also agrees with the theoretical prediction from density wave theory for the assumption of a flat rotation curve in the inner disk. For galaxies with rising rotation curves, the OLR of the (stellar) bar would be spaced farther from its edge (Muñoz-Mateos et al. 2013). ${ }^{27}$

Galaxies with bars are very common in the local universe, with almost two-thirds of nearby galaxies classified as barred in infrared images that trace the stellar population (e.g., de Vaucouleurs et al. 1991; Knapen et al. 2000; Whyte et al. 2002; Laurikainen et al. 2004; Menéndez-Delmestre et al. 2007; Buta et al. 2015; Laine et al. 2016). The decline in the barred fraction of disk galaxies from $f_{\text {bar }} \sim 0.65$ at $z=0$ to $f_{\text {bar }}<0.2$ at $z=0.84$ (Sheth et al. 2008) is almost exclusively in the lowermass $\left(M_{*}=10^{10-11} M_{\odot}\right)$, later-type, and bluer galaxies, potentially due to their dynamically hotter disks (Sheth et al. 2012). In more massive, dynamically colder disks, studies have shown that bars can form out to high redshift $(z \sim 1-2$; Jogee et al. 2004; Simmons et al. 2014). While bars can occur without an interaction, the tidal forces induced by interactions have long been suspected to play a role in bar formation (e.g., Elmegreen et al. 1990; Athanassoula \& Misiriotis 2002), particularly for massive galaxies (Méndez-Abreu et al. 2012), and bars and rings are frequently found together in local interacting systems. Thus, if this interpretation is correct, this could be another indication of interaction-induced substructure in these SMGs. Indeed, the presence of a bar itself would indicate an unstable disk, i.e., a Toomre stability parameter

$$
Q=\frac{\sigma_{\mathrm{r}} \kappa}{\pi \mathrm{G} \Sigma_{\text {disk }}}<1,
$$

where $\sigma_{\mathrm{r}}$ is the one-dimensional velocity dispersion, $\kappa$ is the epicyclic frequency, and $\Sigma_{\text {disk }}$ is the surface density of the disk. Here we assume that the gas disk dominates over the stellar component. Taking the epicyclic frequency appropriate for a flat rotation curve $\left(\kappa=\sqrt{2} V_{\max } / R\right.$ with an assumed $V_{\max }=300 \mathrm{~km} \mathrm{~s}^{-1}$ as typical for SMGs; Bothwell et al. 2013), taking the radius as the HWHM of the ALMA $870 \mu \mathrm{m}$ continuum emission along the major axis, and again estimating the gas surface density from the global $\Sigma_{\text {SFR }}$, we derive upper limits for the one-dimensional velocity dispersion of the potentially barred sources of $\sigma_{\mathrm{r}} \lesssim 70-160 \mathrm{~km} \mathrm{~s}^{-1}$ (Table 5). For these three sources, these Toomre-based upper limits are consistent, albeit marginally, with the lower limits derived from equating the sizes of the largest "clumps" observed to the Jeans length.

If we are indeed observing bar+ring and spiral arm morphologies in some of the sources, we note that the velocity fields would have crossing orbits that would allow efficient loss of angular momentum and collisionally induced star formation.

\footnotetext{
26 Although we note that the morphological characteristics of the bar region of galaxies are strongly influenced by properties of the ISM that may differ at high redshift, such as gas fraction (Athanassoula et al. 2013).

27 Note also that, contrary to the long-standing belief, recent hydrodynamical simulations show that the presence of a stellar bar does not imply that baryons dominate gravitationally in that region (Marasco et al. 2018).
}

These nonaxisymmetric structures (particularly bars) force the gas streams to cross and shock, increasing star formation efficiency and allowing for net angular momentum loss (e.g., Hopkins \& Quataert 2011). The observations presented here may therefore be uncovering the detailed physical mechanisms that result in the very high SFRs measured for SMGs. Ultimately, high-resolution kinematic information is necessary to test the various physical interpretations and confirm the values of the relevant parameters discussed above.

\section{Summary}

We have presented high-fidelity 0 ".07 imaging of the $870 \mu \mathrm{m}$ continuum emission in six luminous galaxies $(z=1.5-4.9)$ from the ALESS SMG survey, allowing us to map the rest-frame FIR emission on $\sim 500 \mathrm{pc}$ scales. Our findings are the following:

1. We report evidence for robust subkiloparsec structure on underlying exponential disks. These structures have deconvolved sizes of $\lesssim 0.5-1 \mathrm{kpc}$. They collectively make up $\sim 2 \%-20 \%$ of the total continuum emission from a given galaxy, indicating that they are not the dominant sites of star formation (assuming a constant dust temperature).

2. We observe no correlation between these structures and those seen in lower-resolution HST imaging, which is extended on larger scales. This comparison suggests that we may be probing the heavily dust-obscured cores of more extended systems.

3. The large-scale morphologies of the structures show (1) clear curvature in the inner $\sim 5-10 \mathrm{kpc}$ for two galaxies (ALESS 112.1 and 15.1) and (2) pairs of clump-like structures bracketing elongated nuclear emission in the three sources that appear to be the most edge-on (ALESS 15.1, 17.1, and 76.1). These observations are suggestive of (1) spiral arms and (2) bars and star-forming rings in inclined disks. The ratio of the "ring" and "bar" radii $(1.9 \pm 0.3)$ is consistent with local galaxies, lending support to this interpretation. The presence of such features may be an indication of tidal disturbances in these systems.

4. We use our high-resolution $870 \mu \mathrm{m}$ imaging to create maps of the SFR density $\left(\Sigma_{\mathrm{SFR}}\right)$ on $\sim 500$ pc scales within the sources, finding peak values that range from $\sim 40$ to $600 M_{\odot} \mathrm{yr}^{-1} \mathrm{kpc}^{-2}$ between sources. We trace this large range in peak $\Sigma_{\mathrm{SFR}}$ back to different galaxy-integrated physical conditions (dust luminosities and temperatures) in the galaxies.

5. Compared to a sample of local U/LIRGs, the SMGs appear to be able to sustain high (galaxy-averaged) rates of star formation over much larger physical scales. However, even on 500 pc scales, they do not exceed the Eddington limit set by radiation pressure on dust. The peak $\Sigma_{\text {SFR }}$ values measured are consistent with those seen in U/LIRGs with similar (total) sizes. As local U/LIRGs can achieve even higher $\Sigma_{\text {SFR }}$ values on smaller physical scales than observable in the SMGs, this may indicate that higher-resolution $(<500$ pc FWHM) observations would be necessary to observe super-Eddington star formation in typical SMGs.

Further observations are required to verify the results presented here. In particular, resolved multifrequency continuum mapping with ALMA is necessary to constrain the variation in dust temperature within the sources (which would affect the 
derived $\Sigma_{\mathrm{SFR}}$ maps), and a larger sample size is important for moving beyond the handful of submillimeter-brightest sources studied here. The striking comparison with the HST imaging highlights the need for high-resolution, near-IR imaging of such dusty targets, such as will become possible with JWST. Finally, high-resolution kinematics are key for confirming the existence of nonaxisymmetric structures within inclined disks. If confirmed by kinematics, the presence of bars would imply that the galaxies have flat rotation curves and Toomre-unstable disks $(Q<1)$. The implied one-dimensional velocity dispersions $\left(\sigma_{\mathrm{r}} \lesssim 70-160 \mathrm{~km} \mathrm{~s}^{-1}\right)$ would be marginally consistent with the lower limits suggested from equating the sizes of the largest clump-like structures observed to the Jeans length. Finally, such nonaxisymmetric structures would provide a mechanism for net angular momentum loss and efficient star formation, helping to explain the very high SFRs measured in SMGs.

The authors wish to thank the anonymous referee for helpful comments that improved this paper. We also thank Sharon Meidt, Arjen van der Wel, Aaron Evans, Francoise Combes, Rob Ivison, and Karin Sandstrom for useful discussions and advice. J.H. and M.R. acknowledge support of the VIDI research program with project No. 639.042.611, which is (partly) financed by the Netherlands Organization for Scientific Research (NWO). I.R.S. acknowledges support from STFC (ST/P000541/1) and the ERC Advanced Grant DUSTYGAL (321334). E.d.C. gratefully acknowledges the Australian Research Council for funding support as the recipient of a Future Fellowship (FT150100079). H.D. acknowledges financial support from the Spanish Ministry of Economy and Competitiveness (MINECO) under the 2014 Ramón y Cajal program MINECO RYC-2014-15686. J.L.W. acknowledges support from an STFC Ernest Rutherford Fellowship (ST/ P004784/1 and ST/P004784/2). This paper makes use of the following ALMA data: ADS/JAO.ALMA\#2016.1.00048.S and ADS/JAO.ALMA \#2012.1.00307.S. ALMA is a partnership of ESO (representing its member states), NSF (USA), and NINS (Japan), together with NRC (Canada), NSC and ASIAA (Taiwan), and KASI (Republic of Korea), in cooperation with the Republic of Chile. The Joint ALMA Observatory is operated by ESO, AUI/NRAO, and NAOJ. The National Radio Astronomy Observatory is a facility of the National Science Foundation operated under cooperative agreement by Associated Universities, Inc.

\section{ORCID iDs}

\section{J. A. Hodge (1) https://orcid.org/0000-0001-6586-8845}

I. Smail (i) https://orcid.org/0000-0003-3037-257X

F. Walter (ib https://orcid.org/0000-0003-4793-7880

E. da Cunha (10) https://orcid.org/0000-0001-9759-4797

A. M. Swinbank (1) https://orcid.org/0000-0003-1192-5837

M. Rybak (i) https://orcid.org/0000-0002-1383-0746

B. Venemans (iD https://orcid.org/0000-0001-9024-8322

W. N. Brandt (iD https://orcid.org/0000-0002-0167-2453

Chian-Chou Chen (iD https://orcid.org/0000-0002-3805-0789

H. Dannerbauer (iD https://orcid.org/0000-0001-7147-3575

R. Decarli (i) https://orcid.org/0000-0002-2662-8803

T. R. Greve (iD https://orcid.org/0000-0002-2554-1837

K. K. Knudsen (iD https://orcid.org/0000-0002-7821-8873

K. M. Menten (10) https://orcid.org/0000-0001-6459-0669

E. Schinnerer (ib https://orcid.org/0000-0002-3933-7677

J. M. Simpson (ib https://orcid.org/0000-0002-8521-1995
P. van der Werf (ib https://orcid.org/0000-0001-5434-5942

A. Weiss (iD https://orcid.org/0000-0003-4678-3939

\section{References}

Alexander, D. M., Bauer, F. E., Chapman, S. C., et al. 2005, ApJ, 632, 736 Andrews, B. H., \& Thompson, T. A. 2011, ApJ, 727, 97

Athanassoula, E. 1992, MNRAS, 259, 328

Athanassoula, E. 2003, MNRAS, 341, 1179

Athanassoula, E., Machado, R. E. G., \& Rodionov, S. A. 2013, MNRAS, 429, 1949

Athanassoula, E., \& Misiriotis, A. 2002, MNRAS, 330, 35

Barcos-Muñoz, L., Leroy, A. K., Evans, A. S., et al. 2017, ApJ, 843, 117

Bekki, K., \& Shioya, Y. 2000, A\&A, 362, 97

Blain, A. W., Smail, I., Ivison, R. J., et al. 2002, PhR, 369, 111

Bothwell, M. S., Smail, I., Chapman, S. C., et al. 2013, MNRAS, 429, 3047

Bottema, R. 2003, MNRAS, 344, 358

Briggs, D. S., Schwab, F. R., \& Sramek, R. A. 1999, in ASP Conf. Ser. 180, Synthesis Imaging in Radio Astronomy II, ed. G. B. Taylor, C. L. Carilli, \& R. A. Perley (San Francisco, CA: ASP), 127

Buta, R. 1986, ApJS, 61, 609

Buta, R. 1995, ApJS, 96, 39

Buta, R., \& Combes, F. 1996, FCPh, 17, 95

Buta, R. J., Sheth, K., Athanassoula, E., et al. 2015, ApJS, 217, 32

Calistro Rivera, G., Hodge, J. A., Smail, I., et al. 2018, ApJ, 863, 56

Cañameras, R., Nesvadba, N., Kneissl, R., et al. 2017, A\&A, 604, A117

Casey, C. M., Narayanan, D., \& Cooray, A. 2014, PhR, 541, 45

Chabrier, G. 2003, ApJL, 586, L133

Chen, C.-C., Hodge, J. A., Smail, I., et al. 2017, ApJ, 846, 108

Chen, C.-C., Smail, I., Swinbank, A. M., et al. 2015, ApJ, 799, 194

Cornwell, T. J. 2008, ISTSP, 2, 793

da Cunha, E., Charlot, S., \& Elbaz, D. 2008, MNRAS, 388, 1595

da Cunha, E., Walter, F., Smail, I. R., et al. 2015, ApJ, 806, 110

Daddi, E., Dickinson, M., Morrison, G., et al. 2007, ApJ, 670, 156

Danielson, A. L. R., Swinbank, A. M., Smail, I., et al. 2017, ApJ, 840, 78

De Breuck, C., Williams, R. J., Swinbank, M., et al. 2014, A\&A, 565, A59

de Vaucouleurs, G., de Vaucouleurs, A., Corwin, H. G., Jr., et al. 1991, Third

Reference Catalogue of Bright Galaxies. Volume I: Explanations and

References. Volume II: Data for Galaxies between $0^{\mathrm{h}}$ and $12^{\mathrm{h}}$. Volume III:

Data for Galaxies between $12^{\mathrm{h}}$ and $24^{\mathrm{h}}$ (New York: Springer)

Dekel, A., Sari, R., \& Ceverino, D. 2009, ApJ, 703, 785

Dobbs, C., \& Baba, J. 2014, PASA, 31, e035

Elbaz, D., Leiton, R., Nagar, N., et al. 2018, A\&A, 616, A110

Elmegreen, D. M., \& Elmegreen, B. G. 2014, ApJ, 781, 11

Elmegreen, D. M., Elmegreen, B. G., \& Bellin, A. D. 1990, ApJ, 364, 415

Engelbracht, C. W., Hunt, L. K., Skibba, R. A., et al. 2010, A\&A, 518, L56

Förster Schreiber, N. M., Shapley, A. E., Genzel, R., et al. 2011, ApJ, 739, 45 Fujimoto, S., Ouchi, M., Kohno, K., et al. 2018, ApJ, 861, 7

Gaia Collaboration, Brown, A. G. A., Vallenari, A., et al. 2016a, A\&A, 595, A2

Gaia Collaboration, Prusti, T., de Bruijne, J. H. J., et al. 2016b, A\&A, 595, A1 Gajda, G., Łokas, E. L., \& Athanassoula, E. 2017, ApJ, 842, 56 Galametz, M., Kennicutt, R. C., Albrecht, M., et al. 2012, MNRAS, 425, 763 Grogin, N. A., Kocevski, D. D., Faber, S. M., et al. 2011, ApJS, 197, 35 Gullberg, B., Swinbank, A. M., Smail, I., et al. 2018, ApJ, 859, 12

Guo, Y., Ferguson, H. C., Bell, E. F., et al. 2015, ApJ, 800, 39

Guo, Y., Giavalisco, M., Ferguson, H. C., Cassata, P., \& Koekemoer, A. M. 2012, ApJ, 757, 120

Hainline, L. J., Blain, A. W., Smail, I., et al. 2011, ApJ, 740, 96

Hatsukade, B., Tamura, Y., Iono, D., et al. 2015, PASJ, 67, 93

Herrera-Endoqui, M., Díaz-García, S., Laurikainen, E., \& Salo, H. 2015, A\&A, 582, A86

Hodge, J. A., Carilli, C. L., Walter, F., et al. 2012, ApJ, 760, 11

Hodge, J. A., Karim, A., Smail, I., et al. 2013, ApJ, 768, 91

Hodge, J. A., Riechers, D., Decarli, R., et al. 2015, ApJL, 798, L18

Hodge, J. A., Swinbank, A. M., Simpson, J. M., et al. 2016, ApJ, 833, 103 Hopkins, P. F., \& Quataert, E. 2011, MNRAS, 415, 1027

Hoyle, B., Masters, K. L., Nichol, R. C., et al. 2011, MNRAS, 415, 3627

Ikarashi, S., Ivison, R. J., Caputi, K. I., et al. 2015, ApJ, 810, 133

Iono, D., Yun, M. S., Aretxaga, I., et al. 2016, ApJL, 829, L10

Jogee, S., Barazza, F. D., Rix, H.-W., et al. 2004, ApJL, 615, L105

Kenney, J. D. P., \& Lord, S. D. 1991, ApJ, 381, 118

Kenney, J. D. P., Wilson, C. D., Scoville, N. Z., Devereux, N. A., \& Young, J. S. 1992, ApJL, 395, L79

Kennicutt, R. C., Jr. 1998, ARA\&A, 36, 189 
Klaas, U., Nielbock, M., Haas, M., Krause, O., \& Schreiber, J. 2010, A\&A, 518, L44

Knapen, J. H., Shlosman, I., \& Peletier, R. F. 2000, ApJ, 529, 93

Koekemoer, A. M., Faber, S. M., Ferguson, H. C., et al. 2011, ApJS, 197, 36

Koprowski, M. P., Dunlop, J. S., Michałowski, M. J., et al. 2016, MNRAS, 458, 4321

Kormendy, J. 1979, ApJ, 227, 714

Kormendy, J., \& Norman, C. A. 1979, ApJ, 233, 539

Kyziropoulos, P. E., Efthymiopoulos, C., Gravvanis, G. A., \& Patsis, P. A. 2016, MNRAS, 463, 2210

Laine, J., Laurikainen, E., \& Salo, H. 2016, A\&A, 596, A25

Laird, E. S., Nandra, K., Pope, A., \& Scott, D. 2010, MNRAS, 401, 2763

Lang, M., Holley-Bockelmann, K., \& Sinha, M. 2014, ApJL, 790, L33

Laurikainen, E., Salo, H., Athanassoula, E., et al. 2013, MNRAS, 430, 3489

Laurikainen, E., Salo, H., \& Buta, R. 2004, ApJ, 607, 103

Law, D. R., Shapley, A. E., Steidel, C. C., et al. 2012, Natur, 487, 338

Lindblad, P. A. B., Lindblad, P. O., \& Athanassoula, E. 1996, A\&A, 313, 65

Luo, B., Brandt, W. N., Xue, Y. Q., et al. 2017, ApJS, 228, 2

Madau, P., \& Dickinson, M. 2014, ARA\&A, 52, 415

Mandelker, N., Dekel, A., Ceverino, D., et al. 2014, MNRAS, 443, 3675

Marasco, A., Oman, K. A., Navarro, J. F., Frenk, C. S., \& Oosterloo, T. 2018, MNRAS, 476, 2168

Marinova, I., \& Jogee, S. 2007, ApJ, 659, 1176

Méndez-Abreu, J., Sánchez-Janssen, R., Aguerri, J. A. L., Corsini, E. M., \& Zarattini, S. 2012, ApJL, 761, L6

Menéndez-Delmestre, K., Sheth, K., Schinnerer, E., Jarrett, T. H., \& Scoville, N. Z. 2007, ApJ, 657, 790

Michałowski, M. J., Hayward, C. C., Dunlop, J. S., et al. 2014, A\&A, 571, A75

Muñoz-Mateos, J. C., Sheth, K., Gil de Paz, A., et al. 2013, ApJ, 771, 59

Murray, N. 2011, ApJ, 729, 133

Murray, N., Quataert, E., \& Thompson, T. A. 2005, ApJ, 618, 569

Murray, N., \& Rahman, M. 2010, ApJ, 709, 424

Noeske, K. G., Weiner, B. J., Faber, S. M., et al. 2007, ApJL, 660, L43

Noguchi, M. 1987, MNRAS, 228, 635

Oh, S. H., Kim, W.-T., Lee, H. M., \& Kim, J. 2008, ApJ, 683, 94

Oteo, I., Zwaan, M. A., Ivison, R. J., Smail, I., \& Biggs, A. D. 2017, ApJ, 837, 182

Peng, C. Y., Ho, L. C., Impey, C. D., \& Rix, H.-W. 2002, AJ, 124, 266

Peng, C. Y., Ho, L. C., Impey, C. D., \& Rix, H.-W. 2010, AJ, 139, 2097

Pérez, I., Aguerri, J. A. L., \& Méndez-Abreu, J. 2012, A\&A, 540, A103

Pettitt, A. R., \& Wadsley, J. W. 2018, MNRAS, 474, 5645

Planck Collaboration, Ade, P. A. R., Aghanim, N., et al. 2016, A\&A, 594, A13

Pohlen, M., Cortese, L., Smith, M. W. L., et al. 2010, A\&A, 518, L72
Rautiainen, P., \& Salo, H. 2000, A\&A, 362, 465

Sanders, D. B., Mazzarella, J. M., Kim, D.-C., Surace, J. A., \& Soifer, B. T. 2003, AJ, 126, 1607

Sanders, R. H., \& Tubbs, A. D. 1980, ApJ, 235, 803

Schwarz, M. P. 1981, ApJ, 247, 77

Scoville, N. 2003, JKAS, 36, 167

Scoville, N., Aussel, H., Sheth, K., et al. 2014, ApJ, 783, 84

Scoville, N., Lee, N., Vanden Bout, P., et al. 2017, ApJ, 837, 150

Scoville, N., Sheth, K., Aussel, H., et al. 2016, ApJ, 820, 83

Scoville, N. Z. 2013, in Evolution of Star Formation and Gas, ed. J. Falcón-Barroso \& J. H. Knapen (Cambridge: Cambridge Univ. Press), 491

Sellwood, J. A., \& Wilkinson, A. 1993, RPPh, 56, 173

Seo, W.-Y., Kim, W.-T., Kwak, S., et al. 2019, ApJ, 872, 5

Sheth, K., Elmegreen, D. M., Elmegreen, B. G., et al. 2008, ApJ, 675, 1141

Sheth, K., Melbourne, J., Elmegreen, D. M., et al. 2012, ApJ, 758, 136

Simmons, B. D., Melvin, T., Lintott, C., et al. 2014, MNRAS, 445, 3466

Simpson, J. M., Smail, I., Swinbank, A. M., et al. 2015, ApJ, 799, 81

Simpson, J. M., Smail, I., Swinbank, A. M., et al. 2017, ApJ, 839, 58

Simpson, J. M., Swinbank, A. M., Smail, I., et al. 2014, ApJ, 788, 125

Speagle, J. S., Steinhardt, C. L., Capak, P. L., \& Silverman, J. D. 2014, ApJS, 214,15

Swinbank, A. M., Dye, S., Nightingale, J. W., et al. 2015, ApJL, 806, L17

Swinbank, A. M., Simpson, J. M., Smail, I., et al. 2014, MNRAS, 438, 1267

Tacconi, L. J., Genzel, R., Neri, R., et al. 2010, Natur, 463, 781

Thompson, T. A., Quataert, E., \& Murray, N. 2005, ApJ, 630, 167

Tomczak, A. R., Quadri, R. F., Tran, K.-V. H., et al. 2016, ApJ, 817, 118

Tremaine, S., \& Weinberg, M. D. 1984, ApJL, 282, L5

Utomo, D., Chiang, I.-D., Leroy, A. K., Sandstrom, K. M., \& Chastenet, J. 2019, ApJ, 874, 141

Walter, F., Decarli, R., Carilli, C. L., et al. 2012, Natur, 486, 233

Wang, S. X., Brandt, W. N., Luo, B., et al. 2013, ApJ, 778, 179

Weinberg, M. D. 1985, MNRAS, 213, 451

Weiner, B. J., Sellwood, J. A., \& Williams, T. B. 2001, ApJ, 546, 931

Weinzirl, T., Jogee, S., Khochfar, S., Burkert, A., \& Kormendy, J. 2009, ApJ, 696,411

Whitaker, K. E., Franx, M., Leja, J., et al. 2014, ApJ, 795, 104

Whitaker, K. E., van Dokkum, P. G., Brammer, G., \& Franx, M. 2012, ApJL, 754, L29

Whyte, L. F., Abraham, R. G., Merrifield, M. R., et al. 2002, MNRAS, 336, 1281

Yuan, T., Richard, J., Gupta, A., et al. 2017, ApJ, 850, 61 